\title{
LIMIT LAW FOR THE COVER TIME OF A RANDOM WALK ON A BINARY TREE
}

\author{
AMIR DEMBO JAY ROSEN OFER ZEITOUNI
}

\begin{abstract}
Let $\mathcal{T}_{n}$ denote the binary tree of depth $n$ augmented by an extra edge connected to its root. Let $\mathcal{C}_{n}$ denote the cover time of $\mathcal{T}_{n}$ by simple random walk. We prove that $\sqrt{\mathcal{C}_{n} 2^{-(n+1)}}-m_{n}$ converges in distribution as $n \rightarrow \infty$, where $m_{n}$ is an explicit constant, and identify the limit.
\end{abstract}

\section{INTRODUCTION}

We introduce in this section notation and our main results, provide background, and give a road map for the rest of the paper.

1.1. Notation and main result. Let $\mathcal{T}_{n}$ denote the binary tree of depth $n$, whose only vertex of degree 2 is attached to an extra vertex $\rho$, called the ROOT. The COVER TIME $\mathcal{C}_{n}$ of $\mathcal{T}_{n}$ is the number of steps of a (discrete time) simple random walk started at $\rho$, till visiting all vertices of $\mathcal{T}_{n}$. We write SRW for such a random walk. The main result of this paper is the following theorem, which gives convergence in law of a (normalized) version of the cover time $\mathcal{C}_{n}$.

Theorem 1.1. Let $\mathcal{C}_{n}^{\prime}:=2^{-(n+1)} \mathcal{C}_{n}$, and set

$$
m_{n}:=\rho_{n} n, \quad \rho_{n}:=c_{*}-\frac{\log n}{c_{*} n}, \quad c_{*}=\sqrt{2 \log 2} .
$$

There exist a random variable $X_{\infty}^{\prime}>0$ and $\alpha_{*}>0$ finite, so that, for any fixed $y \in \mathbb{R}$,

$$
\lim _{n \rightarrow \infty} \mathbb{P}\left(\sqrt{\mathcal{C}_{n}^{\prime}}-m_{n} \leq y\right)=\mathbb{E}\left(\exp \left\{-\alpha_{*} X_{\infty}^{\prime} \mathrm{e}^{-c_{*} y}\right\}\right):=\mathbb{P}\left(Y_{\infty}^{\prime} \leq y\right) .
$$

That is, the normalized cover time $\sqrt{\mathcal{C}_{n}^{\prime}}-m_{n}$ converges in distribution to $Y_{\infty}^{\prime}$, a standard Gumbel random variable shifted by $\log \left(\alpha_{*} X_{\infty}^{\prime}\right)$ then scaled by $1 / c_{*}$. See Lemma 1.3 for a description of the random variable $X_{\infty}^{\prime}$ in terms of the limit of a derivative martingale.

As is often the case, most of the work in proving a statement such as Theorem 1.1 involves the control of certain excursion counts. We now introduce

Date: June 16, 2019.

2010 Mathematics Subject Classification. 60J80; 60J85; 60G50.

Key words and phrases. Cover time. Binary tree. Barrier estimates.

Amir Dembo was partially supported by NSF grant DMS-1613091.

Jay Rosen was partially supported by the Simons Foundation.

Ofer Zeitouni was supported by the ERC advanced grant LogCorFields. 
notation in order to describe these. Let $V_{j}$ denote the set of vertices of $\mathcal{T}_{n}$ at level $j$, with $V_{-1}=\{\rho\}$. For each $v \in V_{n}$ let $v(j)$ denote the ancestor of $v$ at level $j \leq n$, i.e. the unique vertex in $V_{j}$ on the geodesic connecting $v$ and $\rho$. In particular, $v(-1)$ is the root $\rho$.

Next, for each $v \in V_{n}$ let

$$
T_{v, j}^{s}=\#\left\{\text { excursions from } v(j-1) \text { to } v(j) \text { made by the sRW on } \mathcal{T}_{n}\right.
$$

$$
\text { prior to completing its first } s \text { excursions from the root } \rho \text { \}. }
$$

Setting

$$
t_{v, n}^{*}=\inf \left\{s \in \mathbb{Z}_{+}: T_{v, n}^{s} \neq 0\right\},
$$

for the number of excursions to the root by the SRW till reaching $v$, we consider the corresponding excursion cover time of $V_{n}$,

$$
t_{n}^{*}:=\sup _{v \in V_{n}}\left\{t_{v, n}^{*}\right\} .
$$

Our main tool in proving Theorem 1.1 is a generalization (see Proposition 2.2 below), of the following theorem concerning $t_{n}^{*}$.

Theorem 1.2. With notation as in Theorem 1.1,

$$
\sqrt{2 t_{n}^{*}}-m_{n} \stackrel{\text { dist }}{\Longrightarrow} Y_{\infty} \quad \text { as } \quad n \rightarrow \infty
$$

where $Y_{\infty}:=Y_{\infty}^{\prime}-\bar{g}_{\infty}$ for a standard Gaussian random variable $\bar{g}_{\infty}$, independent of $Y_{\infty}^{\prime}$. Alternatively, for some random variable $X_{\infty}>0$,

$$
\mathbb{P}\left(Y_{\infty} \leq y\right):=\mathbb{E}\left(\exp \left\{-\alpha_{*} X_{\infty} \mathrm{e}^{-c_{*} y}\right\}\right) .
$$

As we will see, most of the technical work in the proof of Theorem 1.2 (or Proposition 2.2), is in obtaining the sharp tail estimates of Theorem 1.4 below.

To describe $X_{\infty}$ and $X_{\infty}^{\prime}$, let $\left\{g_{u}, u \in \mathcal{T}_{\infty}\right\}$ be the standard Gaussian branching random walk (BRW), on the infinite binary tree $\mathcal{T}_{\infty}$. That is, placing i.i.d. standard normal weights on the edges of $\mathcal{T}_{\infty}$, we write $g_{u}$ for the sum of the weights along the geodesic connecting 0 to $u$. We further consider the empirically centered $g_{u}^{\prime}:=g_{u}-\bar{g}_{|u|}$, where

$$
\bar{g}_{k}=2^{-k} \sum_{u^{\prime} \in V_{k}} g_{u^{\prime}}, \quad k \in \mathbb{Z}_{+},
$$

denotes the average of the BRW at level $k$, and set

$$
X_{k}=\sum_{u \in V_{k}}\left(c_{*} k+g_{u}\right) \mathrm{e}^{-c_{*}\left(c_{*} k+g_{u}\right)}, X_{k}^{\prime}=\sum_{u \in V_{k}}\left(c_{*} k+g_{u}^{\prime}\right) \mathrm{e}^{-c_{*}\left(c_{*} k+g_{u}^{\prime}\right)} .
$$

It is not hard to verify that $\left\{X_{k}\right\}$ is a martingale, referred to as the DERIVATIVE MARTINGALE. We then have that

Lemma 1.3. $X_{k}, X_{k}^{\prime}$ and $\bar{g}_{k}$ converge a.s. to positive, finite limits $X_{\infty}, X_{\infty}^{\prime}$ and a standard Gaussian variable $\bar{g}_{\infty}$, independent of $X_{\infty}^{\prime}$, such that

$$
X_{\infty}=X_{\infty}^{\prime} e^{-c_{*} \bar{g}_{\infty}} .
$$


The convergence of $X_{k}$ to $X_{\infty}$ is well known, see e.g. 3] (and, for its first occurrence in terms of limits in branching processes, [24]), and building on it, we easily deduce the corresponding convergence for $X_{k}^{\prime}$.

1.2. Background and related results. Theorem 1.1 is closely related to the recent paper [15, which deals with continuous time SRW, and we wish to acknowledge priority to their work. The proofs however are different - while [15] builds heavily on the isomorphism theorem of [21] to relate directly the occupation time on the tree to the Gaussian free field on the tree which is nothing but the BRW described above Lemma 1.3. our proof is a refinement of [8, Theorem 1.3], where the tightness of the LHS of (1.6) is proved. Our proof, which is based on the strategy for proving convergence in law of the maximum of branching random walk described in [13, was obtained independently of [15], except that in proving that Theorem 1.2 implies Theorem 1.1, we do borrow some ideas from [15]. As motivation to our work, we note that estimates from [8] were instrumental in obtaining the tightness of the (centered) cover time of the two dimensional sphere by $\epsilon$-blowup of Brownian motion, see [9]. We expect that the ideas in the current work will play an important role in improving the tightness result of [9] to convergence in law. We defer this to forthcoming work.

We next put our work in context. The study of the cover time of graphs by SRW has a long history. Early bounds appear in [26], and a general result showing that the cover time is concentrated as soon as it is much longer than the maximal hitting time appears in [4]. A modern general perspective linking the cover time of graphs to Gaussian processes appears in [19], and was refined to sharp concentration in [18] (for many graphs including trees) and [30] (for general graphs). See also [25] for a different perspective on [19]. For the cover time of trees, an exact first order asymptotic appears in [5]. The tightness of $\sqrt{\mathcal{C}_{n}^{\prime}}$ around an implicit constant was derived by analytic methods in [14], and, following the identification of the logarithmic correction in $m_{n}$ [20], its $O(1)$ identification appears in [8].

We note that the evaluation of the cover time is but one of many natural questions concerning the process of points with a-typical (local) occupation time, and quite a bit of work has been devoted to this topic. We do not elaborate here and refer the reader to [17, 28, 2]. Particularly relevant to this paper is the recent [1].

It has been recognized for quite some time that the study of the cover time of two dimensional manifolds by Brownian motion (and of the cover time of two dimensional lattices by SRW) is related to a hierarchical structure similar to that appearing in the study of the cover time for trees, see e.g. [16] and, for a recent perspective, [27. A similar hierarchical structure also appears in the study of extremes of the critical Gaussian free field, and in other logarithmically correlated fields appearing e.g. in the study of random matrices. We do not discuss that literature and refer instead to recent surveys offering different perspectives [6, 10, 11, 23, 29]. 
1.3. Structure of the paper. In contrast with [15] the key to our proof of Theorem 1.2 is the following sharp right tail for the excursion cover times.

Theorem 1.4. There exists a finite $\alpha_{*}>0$ such that

$$
\lim _{z \rightarrow \infty} \limsup _{n \rightarrow \infty}\left|z^{-1} \mathrm{e}^{c_{*} z} \mathbb{P}\left(\sqrt{2 t_{n}^{*}}-m_{n}>z\right)-\alpha_{*}\right|=0 .
$$

After quickly dispensing of Lemma 1.3. in Section 2 we obtain Theorem 1.2 out of Theorem 1.4. by adapting the approach of 13 , for the convergence in law of the maximum of BRW. The main difference is that here we have a more general Markov chain (and not merely a sum of i.i.d.-s).

In the short Section 3, which is the only part of this work that parallels the derivation of [15], we deduce Theorem 1.1 out of Theorem 1.2

The bulk of this paper is devoted to the proof of Theorem 1.4, which we establish in Section 4 by a refinement of the approach used in deriving [8, Theorem 1.3]. In doing so, we defer the a-priori bounds we need on certain barrier events, which might be of some independent interest, to Section 5 , where we derive these bounds by refining estimates from [8]. The proof of the main contribution to the tail estimate of Theorem 1.4, as stated in Proposition 4.3, is further deferred to Section 6. There, utilizing the close relation between our Markov chain and the 0-dimensional Bessel process, we get sharper barrier estimates, now up to $(1+o(1))$ factor of the relevant probabilities.

\section{From tail to limit: Lemma 1.3 and Theorem 1.2}

We start by proving the elementary Lemma 1.3. denoting throughout the last common ancestor of $u, u^{\prime} \in \mathcal{T}_{\infty}$ by $w=u \wedge u^{\prime}$. Namely, $w=u(|w|)$ for $|w|=\max \left\{j \geq 0, u(j)=u^{\prime}(j)\right\}$.

Proof of Lemma 1.3. The BRW $\left\{g_{u} ; u \in \mathcal{T}_{k}\right\}$ of Lemma 1.3 is the centered Gaussian random vector having

$$
\operatorname{Cov}\left(g_{u}, g_{u^{\prime}}\right)=\left|u \wedge u^{\prime}\right| \text {. }
$$

Further, the average of the BRW weights on the edges of $\mathcal{T}_{\infty}$ between levels $(k-1)$ and $k$, is precisely $\Delta g_{k}:=\bar{g}_{k}-\bar{g}_{k-1}$ for $\bar{g}_{k}$ of (1.8). With $\left(\Delta g_{k}, k \geq 1\right)$ independent centered Gaussian random variables with $\operatorname{Var}\left(\Delta g_{k}\right)=2^{-k}$, we have that $\bar{g}_{k}$ converges a.s. to the standard Gaussian $\bar{g}_{\infty}:=\sum_{k} \Delta g_{k}$. Next, recall the existence of $w_{k} \rightarrow \infty$ such that, a.s.,

$$
A_{k}:=\left\{c_{*} k+g_{u} \in w_{k}+\left(0,2 c_{*} k\right), \forall u \in V_{k}\right\} \text { occurs for all } k \text { large, }
$$

see e.g. [22, (1.8)]. For $X_{k}$ of (1.9), it follows from [3] that $X_{k} \stackrel{\text { a.s. }}{\rightarrow} X_{\infty} \in(0, \infty)$, while

$$
X_{k}>w_{k} \tilde{X}_{k} \quad \text { on } A_{k}, \quad \text { for } \quad \tilde{X}_{k}:=\sum_{u \in V_{k}} e^{-c_{*}\left(c_{*} k+g_{u}\right)} .
$$

Thus, $\widetilde{X}_{k} \stackrel{\text { a.s. }}{\rightarrow} 0$ as $k \rightarrow \infty$. From the two expressions in (1.9) we have that

$$
X_{k}^{\prime}=\left(X_{k}-\bar{g}_{k} \widetilde{X}_{k}\right) e^{c_{*} \bar{g}_{k}}
$$


which thereby converges a.s. to $X_{\infty}^{\prime}=X_{\infty} e^{c_{*} \bar{g}_{\infty}}$ as claimed in (1.10). Finally, from (2.1) we deduce that for any $u \in V_{k}, k \geq 0$,

$$
\operatorname{Cov}\left(g_{u}, \bar{g}_{k}\right)=2^{-k} \sum_{u^{\prime} \in V_{k}}\left|u \wedge u^{\prime}\right|=\sum_{j=1}^{k}(j-1) 2^{-j}+k 2^{-k}=1-2^{-k} .
$$

This covariance is constant over $u \in V_{k}$, hence $\operatorname{Cov}\left(g_{u}^{\prime}, \bar{g}_{|u|}\right)=0$ for $g_{u}^{\prime}:=$ $g_{u}-\bar{g}_{|u|}$, implying the independence of $\bar{g}_{k}$ and $\left\{g_{u}^{\prime}, u \in V_{k}\right\}$. The latter variables are further independent of the BRW edge weights outside $\mathcal{T}_{k}$, hence of $\bar{g}_{\infty}$. Thus, the random variable $X_{\infty}^{\prime}$, which is measurable on $\sigma\left(g_{u}^{\prime}, u \in \mathcal{T}_{\infty}\right)$, must also be independent of $\bar{g}_{\infty}$.

We next normalize the counts $T_{u, j}^{s}$ of (1.3) and define

$$
\widehat{T}_{u}(s):=\frac{T_{u,|u|}^{s}-s}{\sqrt{2 s}}, \quad \widehat{S}_{k}(s):=2^{-k} \sum_{u \in V_{k}} \widehat{T}_{u}(s),
$$

and get from the CLT for sums of i.i.d. the following relation with the BRW.

Lemma 2.1. For fixed $k$ and the BRW $\left\{g_{u} ; u \in \mathcal{T}_{k}\right\}$ of Lemma 1.3, we have

$$
\begin{array}{r}
\left\{\widehat{T}_{u}(s), u \in \mathcal{T}_{k}\right\} \underset{s \rightarrow \infty}{\stackrel{\text { dist }}{\rightleftharpoons}}\left\{g_{u}, u \in \mathcal{T}_{k}\right\}, \\
\left\{\widehat{T}_{u}(s)-\widehat{S}_{k}(s), u \in V_{k}\right\} \underset{s \rightarrow \infty}{\stackrel{\text { dist }}{\longrightarrow}}\left\{g_{u}^{\prime}, u \in V_{k}\right\} .
\end{array}
$$

Proof. The consecutive excursions to $\rho$ by the sRw on $\mathcal{T}_{n}$ are i.i.d. Hence, $s \mapsto\left\{T_{u,|u|}^{s}, u \in \mathcal{T}_{k}\right\}$ is an $\mathbb{R}^{d}$-valued random walk (with $d$ the finite size of $\left.\mathcal{T}_{k}\right)$. Further, projecting the SRW on $\mathcal{T}_{n}$ to the geodesic from $u$ to $\rho$, yields a symmetric sRW on $\{-1,0, \ldots,|u|\}$. Thus, denoting by $T_{j}$ the number of excursions from $u(j-1)$ to $u \in V_{j}$ during a single excursion to $\rho$, we have that $\mathbb{P}\left(T_{j} \geq 1\right)=\mathrm{p}_{j}:=1 /(j+1)$ (for reaching $u$ before returning to $\rho$ ), and $T_{j}$ conditional on $T_{j} \geq 1$, follows a geometric law of success probability $\mathbb{P}\left(T_{j}=1 \mid T_{j} \geq 1\right)=\mathrm{p}_{j}$. Consequently, for any $j \in[0, k]$,

$$
\mathbb{E}\left(T_{j}\right)=1, \quad \operatorname{Var}\left(T_{j}\right)=\mathbb{E}\left[T_{j}\left(T_{j}-1\right)\right]=\frac{2\left(1-\mathrm{p}_{j}\right)}{\mathrm{p}_{j}}=2 j .
$$

Note that $T_{u,|u|}^{1}$ and $T_{u^{\prime},\left|u^{\prime}\right|}^{1}$ are independent, conditionally on $T_{w,|w|}^{1}$, for $w=$ $u \wedge u^{\prime}$, each having the conditional mean $T_{w,|w|}^{1}$. We thus see that for any $u, u^{\prime} \in \mathcal{T}_{k}$, in view of (2.8),

$$
\operatorname{Cov}\left(T_{u,|u|}^{1}, T_{u^{\prime},\left|u^{\prime}\right|}^{1}\right)=\operatorname{Var}\left(T_{\left|u \wedge u^{\prime}\right|}\right)=2\left|u \wedge u^{\prime}\right| .
$$

Comparing with (2.1), the i.i.d. increments of our $\mathbb{R}^{d}$-valued random walk have the mean vector 1 and covariance matrix which is twice that of the BRW, with (2.6) and (2.7) as immediate consequences of the multivariate CLT. 
Using throughout the notation

$$
s_{n, y}:=\left(m_{n}+y\right)^{2} / 2
$$

for $m_{n}$ of (1.1), we have that

$$
\left\{\sqrt{2 t_{n}^{*}}-m_{n} \leq y\right\}=\left\{t_{n}^{*} \leq s_{n, y}\right\} .
$$

In view of Lemma 2.1, we thus see that Theorem 1.2 is an immediate consequence (for non-random $\tau_{k}(s)=s$ ), of (2.12) in the following lemma. (The additional statement employing (2.13) is utilized in the proof of Theorem 1.1.)

Proposition 2.2. Let $\mathcal{F}_{k}$ denote the $\sigma$-algebra of the $\mathcal{T}_{k}$-projection of the SRW on $\mathcal{T}_{n}$. If $\mathcal{F}_{k}$-measurable $\left\{\tau_{k}(s), s \geq 0\right\}$ are such that

$$
\widehat{\tau}_{k}(s):=\left(\frac{\tau_{k}(s)-s}{\sqrt{2 s}}\right) \underset{s \rightarrow \infty}{\stackrel{p}{\rightarrow}} 0,
$$

then for any fixed $y \in \mathbb{R}$,

$$
\lim _{k \rightarrow \infty} \limsup _{n \rightarrow \infty}\left|\mathbb{P}\left(t_{n}^{*} \leq \tau_{k}\left(s_{n, y}\right)\right)-\mathbb{P}\left(Y_{\infty} \leq y\right)\right|=0 .
$$

Further, replacing (2.11) by

$$
\widehat{\tau}_{k}(s)+\widehat{S}_{k}(s) \underset{s \rightarrow \infty}{\stackrel{p}{\rightarrow}} 0,
$$

leads to (2.12) holding with $Y_{\infty}^{\prime}$ of (1.2) instead of $Y_{\infty}$.

Proof. For a possibly random, $\mathcal{F}_{k}$-measurable $\tau$, we set

$$
\widehat{T}_{u}(\tau ; s):=\frac{T_{u,|u|}^{\tau}-s}{\sqrt{2 s}},
$$

in analogy to $\widehat{T}_{u}(s ; s)=\widehat{T}(s)$ of (2.5). In case $\frac{\tau_{k}(s)}{s} \stackrel{p}{\rightarrow} 1$ as $s \rightarrow \infty$, we get from Donsker's invariance principle that

$$
\widehat{T}_{u}\left(\tau_{k}(s) ; s\right)-\widehat{T}_{u}(s ; s)-\widehat{\tau}_{k}(s) \underset{s \rightarrow \infty}{\stackrel{p}{\longrightarrow}} 0, \quad \forall u \in \mathcal{T}_{k} .
$$

Further,

$$
f_{s}(x):=\sqrt{2(s+\sqrt{2 s} x)}-\sqrt{2 s} \underset{s \rightarrow \infty}{\longrightarrow} x,
$$

uniformly over bounded $x$. Hence, setting

$$
\widetilde{T}_{u}(\tau ; s):=\sqrt{2 T_{u,|u|}^{\tau}}-\sqrt{2 s}=f_{s}\left(\widehat{T}_{u}(\tau ; s)\right),
$$

upon combining Lemma 2.1 and (2.15), we deduce from (2.11) that

$$
\left\{\widetilde{T}_{u}\left(\tau_{k}(s) ; s\right), u \in V_{k}\right\} \underset{s \rightarrow \infty}{\stackrel{\text { dist }}{\Rightarrow}}\left\{g_{u}, u \in V_{k}\right\},
$$

whereas under (2.13) we merely replace $g_{u}$ by $g_{u}^{\prime}$ on the RHS. Proceeding under the assumption (2.11), fix $y \in \mathbb{R}$ and an integer $k \geq 1$, setting

$$
z_{u}^{(n)}:=\sqrt{2 T_{u, k}^{\tau_{k}\left(s_{n, y}\right)}}-m_{n-k}, \quad z_{u}^{(\infty)}:=c_{*} k+g_{u}+y, \quad \forall u \in V_{k},
$$


with $c_{*}$ as in (1.1). For fixed $y$ and $k$, we have, using (2.10), that

$$
\sqrt{2 s_{n, y}}-m_{n-k}-\left(c_{*} k+y\right)=m_{n}-m_{n-k}-c_{*} k=\frac{1}{c_{*}} \log \left(1-\frac{k}{n}\right)_{n \rightarrow \infty} 0 .
$$

Hence from (2.18) at $s=s_{n, y}$ it follows that

$$
\left\{z_{u}^{(n)}, u \in V_{k}\right\} \underset{n \rightarrow \infty}{\stackrel{\text { dist }}{\longrightarrow}}\left\{z_{u}^{(\infty)}, u \in V_{k}\right\} .
$$

In particular, for $X_{k}$ of (1.9) and $\tilde{X}_{k}$ of (2.3) we have that

$$
X_{k}^{(n, y)}:=\sum_{u \in V_{k}} z_{u}^{(n)} e^{-c_{*} z_{u}^{(n)}} \underset{n \rightarrow \infty}{\stackrel{\text { dist }}{\longrightarrow}}\left(X_{k}+y \widetilde{X}_{k}\right) e^{-c_{*} y} .
$$

For any fixed $y \in \mathbb{R}$, we have by (2.2) and (2.20) that

$$
\lim _{k \rightarrow \infty} \varliminf_{n \rightarrow \infty} \mathbb{P}\left(A_{k}^{(n)}\right)=1, \quad A_{k}^{(n)}=\left\{z_{u}^{(n)} \in w_{k}+y+\left(0,2 c_{*} k\right), \forall u \in V_{k}\right\} .
$$

Recalling that $\widetilde{X}_{k} \stackrel{\text { a.s. }}{\rightarrow} 0$ (see the line following (2.3)), and the definition of $Y_{\infty}$ from (1.7), we have in view of (2.21) that for any $\alpha_{k} \rightarrow \alpha_{*}$

$$
\begin{aligned}
\mathbb{P}\left(Y_{\infty} \leq y\right) & =\lim _{k \rightarrow \infty} \mathbb{E}\left[\mathbf{1}_{A_{k}} \exp \left\{-\alpha_{k}\left(X_{k}+y \widetilde{X}_{k}\right) \mathrm{e}^{-c_{*} y}\right\}\right] \\
& =\lim _{k \rightarrow \infty} \varlimsup_{n \rightarrow \infty} \mathbb{E}\left[\mathbf{1}_{A_{k}^{(n)}} \exp \left\{-\alpha_{k} X_{k}^{(n, y)}\right\}\right] \\
& =\lim _{k \rightarrow \infty} \varlimsup_{n \rightarrow \infty} \mathbb{E}\left[\mathbf{1}_{A_{k}^{(n)}} \prod_{u \in V_{k}}\left(1-\alpha_{k} z_{u}^{(n)} e^{-c_{*} z_{u}^{(n)}}\right)\right],
\end{aligned}
$$

where $\varlimsup_{n \rightarrow \infty} f(n)$ stands for bounds given by both $\limsup _{n \rightarrow \infty} f(n)$ and $\liminf _{n \rightarrow \infty} f(n)$, and in the last equality of (2.23) we relied on having

$$
\delta_{k}:=\sup _{n} \mathbf{1}_{A_{k}^{(n)}} \sup _{u \in V_{k}}\left\{\alpha_{k} z_{u}^{(n)} e^{-c_{*} z_{u}^{(n)}}\right\}_{k \rightarrow \infty}^{\longrightarrow} 0,
$$

as well as $e^{-a} \geq 1-a \geq e^{-a(1+\delta)}$ for $a \in[0, \delta \wedge 1 / 2]$. For $u \in V_{k}$ let $V_{n}^{u}=$ $\left\{v \in V_{n}: v(k)=u\right\}$ denote the leaves of the binary sub-tree of $\mathcal{T}_{n}$ of depth $n-k$, emanating from $u$, with $u(k-1)$ acting as its (extra) root. The event $\left\{t_{n}^{*} \leq \tau\right\}$ of the SRW reaching all of $V_{n}$ within its first $\tau$ excursions to $\rho$ is the intersection over $u \in V_{k}$ of the events of reaching all of $V_{n}^{u}$ within the first $T_{u, k}^{\tau}$ excursions of the SRW between $u(k-1)$ and $u$. By the Markov property, for $\mathcal{F}_{k^{-}}$ measurable $\tau$, conditionally on $\mathcal{F}_{k}$ the latter events are mutually independent, of conditional probabilities $\bar{\gamma}_{n-k}\left(T_{u, k}^{\tau}\right)$ for $u \in V_{k}$ and $\bar{\gamma}_{n}(s):=\mathbb{P}\left(t_{n}^{*} \leq s\right)$. Consequently, for $\tau=\tau_{k}\left(s_{n, y}\right)$ we get that

$$
\mathbb{P}\left(t_{n}^{*} \leq \tau \mid \mathcal{F}_{k}\right)=\prod_{u \in V_{k}} \bar{\gamma}_{n-k}\left(T_{u, k}^{\tau}\right)=\prod_{u \in V_{k}}\left(1-\gamma_{n-k}\left(z_{u}^{(n)}\right)\right),
$$

for $\gamma_{n}(z):=\mathbb{P}\left(\sqrt{2 t_{n}^{*}}-m_{n}>z\right)$ and $z_{u}^{(n)}$ of (2.19). Theorem 1.4 and the monotonicity of $z \mapsto \gamma_{n}(z)$ yield that for some $n_{k}<\infty$ and $\alpha_{k}^{( \pm)} \rightarrow \alpha_{*}$,

$$
\alpha_{k}^{(-)} z e^{-c_{*} z} \leq \gamma_{n}(z) \leq \alpha_{k}^{(+)} z e^{-c_{*} z} \quad \forall n \geq n_{k}, \forall z \in w_{k}+y+\left[0,2 c_{*} k\right] .
$$


Under the event $A_{k}^{(n)}$, which is measurable on $\mathcal{F}_{k}$, the latter bounds apply for all $z=z_{u}^{(n)}$. Hence, we get from (2.24) that

$$
\begin{aligned}
& \varliminf_{n \rightarrow \infty} \mathbb{E}\left[\mathbf{1}_{A_{k}^{(n)}} \prod_{u \in V_{k}}\left(1-\alpha_{k}^{(+)} z_{u}^{(n)} e^{-c_{*} z_{u}^{(n)}}\right)\right] \leq \varliminf_{n \rightarrow \infty} \mathbb{P}\left(t_{n}^{*} \leq \tau_{k}\left(s_{n, y}\right) ; A_{k}^{(n)}\right) \\
& \leq \varlimsup_{n \rightarrow \infty} \mathbb{P}\left(t_{n}^{*} \leq \tau_{k}\left(s_{n, y}\right) ; A_{k}^{(n)}\right) \leq \varlimsup_{n \rightarrow \infty} \mathbb{E}\left[\mathbf{1}_{A_{k}^{(n)}} \prod_{u \in V_{k}}\left(1-\alpha_{k}^{(-)} z_{u}^{(n)} e^{-c_{*} z_{u}^{(n)}}\right)\right] .
\end{aligned}
$$

We now establish (2.12), by taking $k \rightarrow \infty$ while utilizing (2.22) and (2.23).

The same argument applies under (2.13), now replacing $g_{u}$ by $g_{u}^{\prime}$ in (2.19), thereby changing $X_{k}, \widetilde{X}_{k}$ and $Y_{\infty}$ in (2.21) and (2.23) , to $X_{k}^{\prime}, \widetilde{X}_{k}^{\prime}$ and $Y_{\infty}^{\prime}$.

\section{EXCURSION COUNTS TO REAL TIME: \\ From Proposition 2.2 To Theorem 1.1}

Theorem 1.1 amounts to showing that for any fixed $y \in \mathbb{R}$ and $\epsilon>0$,

$$
\varlimsup_{n \rightarrow \infty} \mathbb{P}\left(\mathcal{C}_{n}^{\prime} \leq 2 s_{n, y-2 \epsilon}\right) \leq \mathbb{P}\left(Y_{\infty}^{\prime} \leq y\right) \leq \varliminf_{n \rightarrow \infty} \mathbb{P}\left(\mathcal{C}_{n}^{\prime} \leq 2 s_{n, y+2 \epsilon}\right),
$$

where throughout $s_{n, y}:=\left(m_{n}+y\right)^{2} / 2$, as in (2.10). To this end, let

$$
R_{n}^{s}:=2^{-n} \sum_{u \in \mathcal{T}_{n}} T_{u,|u|}^{s} \cdot
$$

The SRW on $\mathcal{T}_{n}$ makes $2^{(n+1)} R_{n}^{s}$ steps during its first $s$ excursions from the root to itself. Thus, $\left\{t_{n}^{*} \leq \tau\right\}=\left\{\mathcal{C}_{n}^{\prime} \leq R_{n}^{\tau}\right\}$, so for any random $t, \tau$,

$$
\mathbb{P}\left(t_{n}^{*} \leq \tau\right)-\mathbb{P}\left(R_{n}^{\tau}>2 t\right) \leq \mathbb{P}\left(\mathcal{C}_{n}^{\prime} \leq 2 t\right) \leq \mathbb{P}\left(t_{n}^{*} \leq \tau\right)+\mathbb{P}\left(R_{n}^{\tau}<2 t\right) .
$$

Considering (3.3) at $t=s_{n, y \pm 2 \epsilon}$, the insufficient concentration of $R_{n}^{\tau}$ at the non-random $\tau=s_{n, y}$ rules out establishing (3.1) directly from Theorem 1.2. We thus follow the approach of [15, Section 9], in employing instead (3.3) for $\tau=\tau_{k}\left(s_{n, y}\right)$ and the $\mathcal{F}_{k}$-measurable

$$
\tau_{k}(s):=\inf \left\{\ell \in \mathbb{Z}_{+} \mid S_{k}^{\ell} \geq s\right\}, \quad S_{k}^{\ell}:=2^{-k} \sum_{u \in V_{k}} T_{u, k}^{\ell} .
$$

Recall that $\mathbb{E}\left[S_{k}^{1}\right]=1$ (see (2.8)), while setting $\bar{\sigma}_{k}^{2}:=\operatorname{Var}\left(\bar{g}_{k}\right)=1-2^{-k}$ (see (2.4)), and comparing (2.1) to (2.9), we arrive at $\operatorname{Var}\left(S_{k}^{1}\right)=2 \bar{\sigma}_{k}^{2}$. Hence, Donsker's invariance principle yields a coupling between the piece-wise linear interpolation $t \mapsto \widehat{S}_{s, k}(t)$ of $\left\{\left(S_{k}^{t}-t\right) / \sqrt{2 s} ; t \in \mathbb{Z}_{+}\right\}$, and a standard Brownian motion $\left\{W_{\theta}\right\}$, such that

$$
\sup _{\theta \in[0,2]}\left|\widehat{S}_{s, k}(\theta s)-\bar{\sigma}_{k} W_{\theta}\right| \underset{s \rightarrow \infty}{\stackrel{p}{\longrightarrow}} 0 .
$$

From (3.4) we see that $S_{k}^{\tau_{k}(s)}-s \geq 0$ is at most the total number of excursions from $V_{k-1}$ to $V_{k}$ made by the sRW started at some $v \in V_{k}$, before hitting the 
root, plus 1 . The latter has exactly the law of $2^{k} S_{k}^{1}$ given $S_{k}^{1}>0$. Thus,

$$
c_{k}:=\sup _{s \geq 0}\left\{\mathbb{E}\left[S_{k}^{\tau_{k}(s)}-s\right]\right\} \leq \frac{\mathbb{E}\left[S_{k}^{1}\right]}{\mathbb{P}\left(S_{k}^{1}>0\right)}<\infty,
$$

and for $\widehat{\tau}_{k}(s)$ defined as in (2.11), one has when $s \rightarrow \infty$, that

$$
\widehat{S}_{s, k}\left(\tau_{k}(s)\right)+\widehat{\tau}_{k}(s)=\frac{S_{k}^{\tau_{k}(s)}-s}{\sqrt{2 s}} \stackrel{p}{\longrightarrow} 0, \quad \theta_{s}:=\frac{\tau_{k}(s)}{s} \stackrel{p}{\longrightarrow} 1 .
$$

In particular, considering (3.5) at $\theta_{s}$, by the continuity of $\theta \mapsto W_{\theta}$

$$
\widehat{S}_{s, k}\left(\theta_{s} s\right)=\bar{\sigma}_{k} W_{\theta_{s}}+o_{p}(1)=\bar{\sigma}_{k} W_{1}+o_{p}(1)=\widehat{S}_{s, k}(s)+o_{p}(1) .
$$

Since $\widehat{S}_{s, k}(s)=\widehat{S}_{k}(s)$ of (2.5), we conclude that $\left\{\tau_{k}(s), s \geq 0\right\}$ of (3.4) satisfy (2.13), and with $\left|2 s_{n, y \pm 2 \epsilon}-2 s_{n, y}\right| \geq 4 \epsilon \sqrt{s_{n, y}}$ for $n$ large enough, we finish the proof of Theorem 1.1 upon showing that for $s=s_{n, y}$ and any fixed $\epsilon>0$,

$$
\lim _{k \rightarrow \infty} \varlimsup_{n \rightarrow \infty} \mathbb{P}\left(\left|R_{n}^{\tau_{k}(s)}-2 s\right| \geq 4 \epsilon \sqrt{s}\right)=0 .
$$

To this end, recall that in view of (2.8) and (3.2)

$$
r_{j}:=\mathbb{E}\left(R_{j}^{1}\right)=2^{-j}\left|\mathcal{T}_{j}\right|=2-2^{-j},
$$

and similarly, by (2.9) and (3.2) we get that

$$
\sigma_{n}^{2}:=\operatorname{Var}\left(R_{n}^{1}\right)=2 \sum_{u, u^{\prime} \in \mathcal{T}_{n}} 2^{-2 n}\left|u \wedge u^{\prime}\right| \leq 4 .
$$

Next, writing in short $\tau=\tau_{k}(s)$, we have for any $n \geq k$, the representation

$$
R_{n}^{\tau}=2^{k-n} R_{k}^{\tau}+r_{n-k} S_{k}^{\tau}+2^{-k} \Delta_{k, n}\left(2^{k} S_{k}^{\tau}\right),
$$

where the random variable $\Delta_{k, n}(\ell)$ is the centered and scaled total time spent by the sRW on $\mathcal{T}_{n}$ below level $k$ during the first $\ell$ excursions from $V_{k-1}$ to $V_{k}$. For fixed $\ell, \Delta_{k, n}(\ell) \stackrel{(d)}{=} R_{n-k}^{\ell}-\mathbb{E}\left(R_{n-k}^{\ell}\right)$ and has variance $\sigma_{n-k}^{2} \ell \leq 4 \ell$. Further, $\Delta_{k, n}\left(2^{k} S_{k}^{\tau}\right)$ conditioned on $\mathcal{F}_{k}$ is distributed as $R_{n-k}^{\ell}-\mathbb{E}\left(R_{n-k}^{\ell}\right)$ with $\ell=2^{k} S_{k}^{\tau}$. Recalling that $R_{k-1}^{\ell}=2\left(R_{k}^{\ell}-S_{k}^{\ell}\right)$ and utilizing (3.6), we thus get by Markov's inequality (conditional on $\mathcal{F}_{k}$ ), that

$$
\delta_{k, n}:=\mathbb{P}\left(\left|R_{n}^{\tau}-2^{k-n-1} R_{k-1}^{\tau}-2 S_{k}^{\tau}\right| \geq \epsilon \sqrt{s}\right) \leq \frac{42^{-k}}{\epsilon^{2}} \frac{\mathbb{E}\left(S_{k}^{\tau}\right)}{s}=\frac{42^{-k}}{\epsilon^{2}}\left(1+\frac{c_{k}}{s}\right),
$$

goes to zero for $s=s_{n, y} \rightarrow \infty$ followed by $k \rightarrow \infty$. Also, by the union bound,

$$
\begin{aligned}
\mathbb{P}\left(\left|R_{n}^{\tau}-2 s\right| \geq 4 \epsilon \sqrt{s}\right) \leq \delta_{k, n}+\mathbb{P}\left(S_{k}^{\tau}-s \geq \epsilon \sqrt{s}\right) & +\mathbb{P}(\tau \geq 2 s) \\
& +\mathbb{P}\left(2^{k-n-1} R_{k-1}^{2 s} \geq \epsilon \sqrt{s}\right) .
\end{aligned}
$$

Next, employing Markov's inequality, we deduce that the last term goes to zero, since $2^{k-n} s r_{k-1} /(\epsilon \sqrt{s}) \rightarrow 0$ for $s=s_{n, y}$ and $n \rightarrow \infty$. By (3.6) and having WHP $\tau=\tau_{k}(s)<2 s$ (see (3.7)), we thus arrive at (3.8) and thereby conclude the proof of Theorem 1.1. 


\section{Sharp Right tail: auxiliary lemmas and proof of Theorem 1.4}

Hereafter we denote by $\mathbb{P}_{s}$ probabilities of events occurring up to the completion of the first $s$ excursions at the root and let $\eta_{v}(j):=\sqrt{2 T_{v, j}^{s}}$ for $v \in V_{k}$, $j \leq k$ and $T_{v, j}^{s}$ of (1.3), with the value of $s$ implicit. For $u \in V_{n^{\prime}}$ where $n^{\prime}:=n-\ell$, and $V_{n}^{u}:=\left\{v \in V_{n}: v\left(n^{\prime}\right)=u\right\}$, let

$$
\eta_{\ell}^{\sharp}(u):=\min _{v \in V_{n}^{u}}\left\{\eta_{v}(n)\right\},
$$

denote the minimal (normalized) occupation time of edges entering leaves of the sub-tree of depth $\ell$ rooted at $u$ (during the first $s$ excursions from the root), abbreviating $\eta_{n}^{\sharp}$ for $\eta_{n}^{\sharp}(0)$. Since

$$
\left\{t_{n}^{*}>s\right\}=\left\{\min _{v \in V_{n}}\left\{T_{v, n}^{s}\right\}=0\right\},
$$

Theorem 1.4 amounts to the claim

$$
\alpha_{*}=\lim _{z \rightarrow \infty} z^{-1} \mathrm{e}^{c_{*} z} \varlimsup_{n \rightarrow \infty} \mathbb{P}_{s_{n, z}}\left(\eta_{n}^{\sharp}=0\right),
$$

for $s_{n, z}$ and $c_{*}$ of (2.10) and (1.1), respectively. Our proof of (4.2) is based on a refinement of the probability estimates of [8, Section 5], intersecting here the event $\left\{\eta_{n}^{\sharp}=0\right\}$ with barrier events involving the (normalized) edge occupation times $\left\{j \mapsto \eta_{v}(j), v \in \mathcal{T}_{n}\right\}$. More precisely, we adapt the strategy of [13, Section 3], by essentially bounding $\mathbb{P}_{s_{n, z}}\left(\eta_{n}^{\sharp}=0\right)$ between the expectations of counts $\Lambda_{n, \ell} \leq \Gamma_{n, \ell}$ for two barrier type events, which are equivalent at the claimed scale of asymptotic growth in $z$ (see Lemma 4.2). Our curved barrier event for $\Gamma_{n, \ell}$ is relaxed enough to deduce that the event $\left\{\Gamma_{n, \ell} \geq 1\right\}$ is for large $n, \ell$, about the same as having $\left\{\eta_{n}^{\sharp}=0\right\}$ (see Lemma 4.1). The straight barrier event for $\Lambda_{n, \ell}$ is strict enough to yield a negligible variance (see Lemma 4.4), so its expectation serves to lower bound $\mathbb{P}_{s_{n, z}}\left(\eta_{n}^{\sharp}=0\right)$. Our claim (4.2) then follows from such a limit for $\mathbb{E}_{s_{n, z}}\left[\Lambda_{n, \ell}\right]$ (which is a consequence of Proposition 4.3). Specifically, for $s=s_{n, z}$ consider the excess edge occupation times, over the barrier

$$
\bar{\varphi}_{n}(j):=\rho_{n}(n-j), \quad j \in\left[0, n^{\prime}\right], \quad n^{\prime}=n-\ell .
$$

In the sequel we show that the main contribution to $\left\{\eta_{n}^{\sharp}=0\right\}$ is due to not covering a sub-tree rooted at some $u \in V_{n^{\prime}}$ while the edge occupation times along the geodesic to $u$ exceed the barrier $\bar{\varphi}_{n}(\cdot)$ of (4.3), with the excess at the edge into $u$ further restricted to

$$
I_{\ell}:=\sqrt{\ell}\left[r_{\ell}^{-1}, r_{\ell}\right], \quad r_{\ell}:=\sqrt{\log \ell} .
$$

To this end, let

$$
\hat{\eta}_{v}(j):=\eta_{v}(j)-\bar{\varphi}_{n}(j)
$$




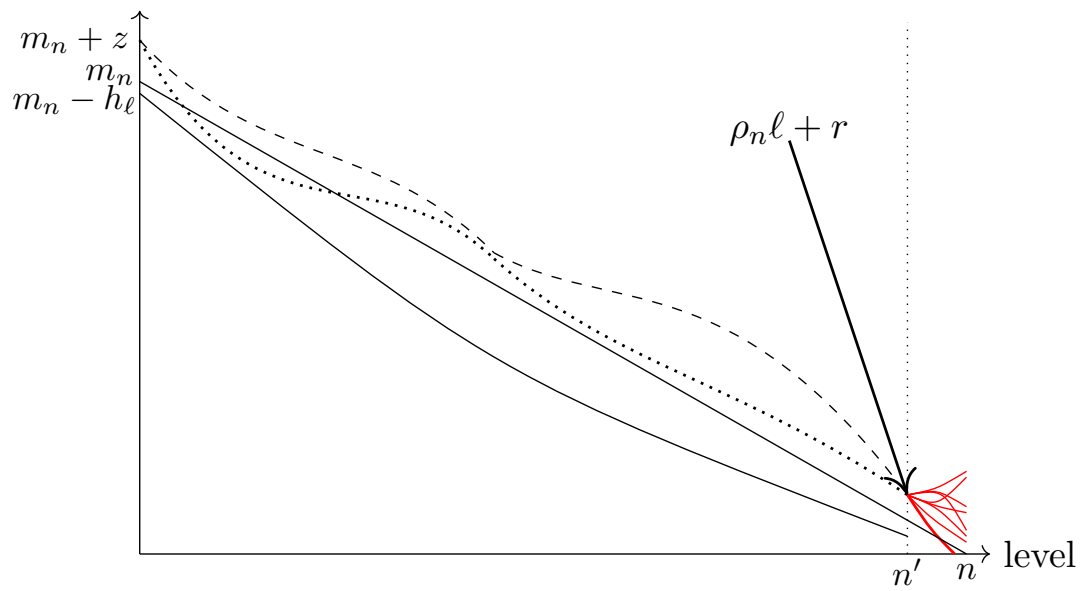

FiguRE 1. Depiction of the events $E_{n, \ell}(u)$ (dashed line) and $F_{n, \ell}(u)$ (dotted line) for some $u \in V_{n^{\prime}}$. In either case, the red paths emanating from level $n^{\prime}=n-\ell$ denote excursion counts corresponding to different children of $u$. Note the curved vs. straight barrier and the excursion count that reaches 0 .

considering for $u \in V_{n^{\prime}}$ the events

$$
E_{n, \ell}(u):=\bigcap_{0 \leq j \leq n^{\prime}}\left\{\hat{\eta}_{u}(j)>0\right\} \bigcap\left\{\hat{\eta}_{u}\left(n^{\prime}\right) \in I_{\ell}, \eta_{\ell}^{\sharp}(u)=0\right\},
$$

and the corresponding counts

$$
\Lambda_{n, \ell}:=\sum_{u \in V_{n^{\prime}}} \mathbf{1}_{E_{n, \ell}(u)} .
$$

See Figure 1 for a pictorial illustration of the event $E_{n, \ell}(u)$. As explained before, aiming first to upper bound $\mathbb{P}_{s_{n, z}}\left(\eta_{n}^{\sharp}=0\right)$, we fix $\delta \in\left(0, \frac{1}{2}\right)$ and for $k \in[1, n], h \in[0, n-k]$, consider the curved, relaxed barriers

$$
\varphi_{n, k, h}(j):=\bar{\varphi}_{n}(j)-\psi_{k, h}(j), \quad j \in[0, k],
$$

using hereafter the notations

$$
\psi_{k, h}(j):=h+j_{k}^{\delta}, \quad j_{k}:=j \wedge(k-j), \quad j \in[0, k] .
$$

We further use the abbreviated notation

$$
\psi_{\ell}(\cdot):=\psi_{n^{\prime}, h_{\ell}}(\cdot), \quad \text { where } \quad h_{\ell}:=\frac{1}{2} \log \ell,
$$

with $n^{\prime}=n-\ell \geq 1$. Replacing the barriers of (4.3) by those of (4.8), we then form the larger counts

$$
\Gamma_{n, \ell}=\sum_{u \in V_{n^{\prime}}} \mathbf{1}_{F_{n, \ell}(u)}
$$




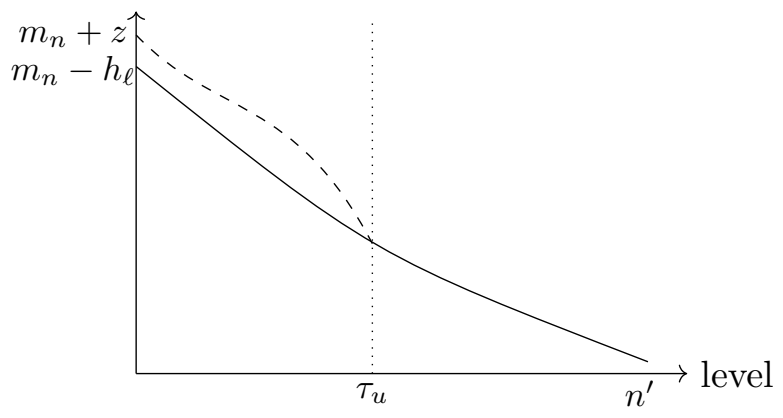

FiguRE 2. Depiction of an event from $G_{n, \ell}$ (dashed line) corresponding to some $u \in V_{n^{\prime}}, n^{\prime}=n-\ell$. Note the curved barrier.

where in terms of (4.1), (4.5) and (4.8), we define for each $u \in V_{n^{\prime}}$

$$
F_{n, \ell}(u):=\bigcap_{0 \leq j \leq n^{\prime}}\left\{\hat{\eta}_{u}(j)+\psi_{\ell}(j)>0\right\} \bigcap\left\{\eta_{\ell}^{\sharp}(u)=0\right\} .
$$

See Figure 1 for a pictorial illustration of the event $F_{n, \ell}(u)$. If $\eta_{n}^{\sharp}=0$, then necessarily $\eta_{\ell}^{\sharp}(u)=0$ for some $u \in V_{n^{\prime}}$ and either $F_{n, \ell}(u)$ occurs (so $\Gamma_{n, \ell} \geq 1$ ), or else the event $G_{n, \ell}:=G_{n, n^{\prime}}\left(h_{\ell}\right)$ must occur, where, see Figure 2 ,

$$
G_{n, k^{\prime}}(h):=\bigcup_{u \in V_{k^{\prime}}} \bigcup_{0 \leq j \leq k^{\prime}}\left\{\hat{\eta}_{u}(j) \leq-\psi_{k^{\prime}, h}(j)\right\} .
$$

Hence, for any $\ell$,

$$
\mathbb{E}_{s_{n, z}}\left[\Gamma_{n, \ell}\right] \geq \mathbb{P}_{s_{n, z}}\left(\Gamma_{n, \ell} \geq 1\right) \geq \mathbb{P}_{s_{n, z}}\left(\eta_{n}^{\sharp}=0\right)-\mathbb{P}_{s_{n, z}}\left(G_{n, \ell}\right) .
$$

Recall that by [8, proof of Corollary 5.4], for some $c^{\prime}>0$ and all $z \geq 1$,

$$
\varliminf_{n \rightarrow \infty} \mathbb{P}_{s_{n, z}}\left(\eta_{n}^{\sharp}=0\right) \geq c^{\prime} z \mathrm{e}^{-c_{*} z},
$$

so our next lemma, which is an immediate consequence of Lemma 5.1 below, shows that the right-most term in (4.14) is negligible.

Lemma 4.1. We have that

$$
\lim _{\ell \rightarrow \infty} \sup _{z \geq 1}\left\{z^{-1} e^{c_{*} z} \varlimsup_{n \rightarrow \infty} \mathbb{P}_{s_{n, z}}\left(G_{n, \ell}\right)\right\}=0 .
$$

Combining (4.14)-(4.16), we arrive at

$$
\varlimsup_{\ell \rightarrow \infty} \varlimsup_{z \rightarrow \infty} \varlimsup_{n \rightarrow \infty} \frac{\mathbb{P}_{s_{n, z}}\left(\eta_{n}^{\sharp}=0\right)}{\mathbb{E}_{s_{n, z}}\left[\Gamma_{n, \ell}\right]} \leq 1 .
$$

Restricting hereafter to $\delta \in\left(0, \frac{1}{6}\right)$ allows us to further show in Section 5.2 the following equivalence of first moments (c.f. (5.41) for why we take $\delta$ small). 
Lemma 4.2. For any $\delta \in\left(0, \frac{1}{6}\right)$ we have that

$$
\lim _{\ell \rightarrow \infty} \varlimsup_{z \rightarrow \infty}\left\{z^{-1} e^{c_{*} z} \varlimsup_{n \rightarrow \infty} \mathbb{E}_{s_{n, z}}\left[\Gamma_{n, \ell}-\Lambda_{n, \ell}\right]\right\}=0 .
$$

Now, from (4.17) and (4.18), we have the upper bound

$$
\varlimsup_{\ell \rightarrow \infty} \varlimsup_{z \rightarrow \infty} \varlimsup_{n \rightarrow \infty} \frac{\mathbb{P}_{s_{n, z}}\left(\eta_{n}^{\sharp}=0\right)}{\mathbb{E}_{s_{n, z}}\left[\Lambda_{n, \ell}\right]} \leq 1 .
$$

For such expected counts with straight barriers, we establish in Section 6.1, using the connection to the 0-Bessel process, the following large $n$ and $z$ asymptotic.

Proposition 4.3. There exists $\alpha_{\ell}>0$ such that

$$
\lim _{\ell \rightarrow \infty} \alpha_{\ell}^{-1}\left\{\varlimsup_{z \rightarrow \infty} z^{-1} \mathrm{e}^{c_{*} z} \varlimsup_{n \rightarrow \infty} \mathbb{E}_{s_{n, z}}\left[\Lambda_{n, \ell}\right]\right\}=1,
$$

where by 4.15) and 4.19), $\lim \inf \left\{\alpha_{\ell}\right\}$ is strictly positive.

As shown in Section 5.3, the barrier event we have added in the definition (4.6) of $E_{n, \ell}(u)$ yields the following tight control on the second moment of $\Lambda_{n, \ell}$.

Lemma 4.4. We have that

$$
\lim _{\ell \rightarrow \infty} \varlimsup_{z \rightarrow \infty}\left\{z^{-1} e^{c_{*} z} \varlimsup_{n \rightarrow \infty} \mathbb{E}_{s_{n, z}}\left[\Lambda_{n, \ell}\left(\Lambda_{n, \ell}-1\right)\right]\right\}=0 .
$$

Note that $\Lambda_{n, \ell} \geq 1$ implies having $\eta_{v}(n)=0$ for some $v \in V_{n}$, that is, having $\eta_{n}^{\sharp}=0$. Hence, with $\Lambda_{n, \ell}$ integer valued, for any choice of $\ell$,

$$
\mathbb{P}_{s_{n, z}}\left(\eta_{n}^{\sharp}=0\right) \geq \mathbb{P}_{s_{n, z}}\left(\Lambda_{n, \ell} \geq 1\right) \geq \mathbb{E}_{s_{n, z}}\left[\Lambda_{n, \ell}\right]-\mathbb{E}_{s_{n, z}}\left[\Lambda_{n, \ell}\left(\Lambda_{n, \ell}-1\right)\right] .
$$

Having a positive $\lim \inf \left\{\alpha_{\ell}\right\}$, the latter bound, together with (4.20) and (4.21), imply that

$$
\varliminf_{\ell \rightarrow \infty} \varliminf_{z \rightarrow \infty} \varliminf_{n \rightarrow \infty} \frac{\mathbb{P}_{s_{n, z}}\left(\eta_{n}^{\sharp}=0\right)}{\mathbb{E}_{s_{n, z}}\left[\Lambda_{n, \ell}\right]} \geq 1
$$

Proof of Theorem 1.4. Comparing first (4.19) to (4.23) and then with (4.20), we conclude that

$$
\lim _{\ell \rightarrow \infty} \alpha_{\ell}^{-1}\left\{\varlimsup_{n \rightarrow \infty}^{\varlimsup^{-1}} z^{c_{*} z} \varlimsup_{n \rightarrow \infty} \mathbb{P}_{s_{n, z}}\left(\eta_{n}^{\sharp}=0\right)\right\}=1 .
$$

Necessarily $\alpha_{\ell} \rightarrow \alpha_{*}$ for which (4.2) holds (with $\alpha_{*}>0$ in view of (4.15) and $\alpha_{*}<\infty$ by [8, Proposition 5.2]). 


\section{BARRIER BOUNDS FOR EXCURSION COUNTS}

We keep the barrier sequences of (4.8) and all other related notation from Section 4. Further, with $\rho_{n} \rightarrow c_{*}>1.1$, see (1.1), WLOG we restrict to $n \geq n_{*} \geq 64$ with $\rho_{n} \geq \rho_{*}=: 1.1$, starting at the following a-priori bound on the events $G_{n, k^{\prime}}(h)$ from (4.13). Recall the notation $s_{n, z}$ of (2.10).

Lemma 5.1. For some $c<\infty$, any $n \geq n_{*}, z, k^{\prime} \geq 1$ and $h \in\left[0, n-k^{\prime}\right]$,

$$
\mathbb{P}_{s_{n, z}}\left(G_{n, k^{\prime}}(h)\right) \leq c(z+h) e^{-c_{*}(z+h)} e^{-(z+h)^{2} /(8 n)} .
$$

Proof of Lemma 4.1. Setting $h=h_{\ell}=\frac{1}{2} \log \ell$ in (5.1), results with

$$
\mathbb{P}_{s_{n, z}}\left(G_{n, \ell}\right) \leq c(z+\log \ell) \ell^{-c_{*} / 2} e^{-c_{*} z} e^{-z^{2} /(8 n)},
$$

so taking $\ell \rightarrow \infty$ establishes (4.16).

Before embarking on the proof of Lemma 5.1, we deduce from it certain useful a-priori tail bounds on the non-covering events $\left\{\eta_{\ell}^{\sharp}=0\right\}$.

Corollary 5.2. For some $c<\infty$ and all $n \geq n_{\star}, z \geq 1$,

$$
\mathbb{P}_{s_{n, z}}\left(\eta_{n}^{\sharp}=0\right) \leq c z e^{-c_{*} z} e^{-z^{2} /(8 n)} .
$$

Further, for some $\hat{\ell}$ finite, any $\hat{\ell} \leq \ell \leq n / \log n$ and $r \geq-h_{\ell}$,

$$
\gamma_{n, \ell}(r):=\mathbb{P}_{\left[\left(\rho_{n} \ell+r\right)^{2} / 2\right]}\left(\eta_{\ell}^{\sharp}=0\right) \leq c \ell^{-1}(r+\log \ell) e^{-c_{*} r} e^{-r^{2} /(8 \ell)} .
$$

Proof. The event $\eta_{n}^{\sharp}=0$ amounts to $\eta_{v}(n)=0$ for some $v \in V_{n}$. With $\varphi_{n, n, 0}(n)=0$ (see (4.8)-(4.9) ), this implies that $\eta_{v}(n) \leq \varphi_{n, n, 0}(n)$ and consequently that $G_{n, n}(0)$ also occurs (take $j=k^{\prime}=n$ in (4.13)). That is $\left\{\eta_{n}^{\sharp}=0\right\} \subseteq G_{n, n}(0)$, so the bound (5.3) follows from (5.1). Proceeding to prove (5.4) , setting $\hat{\ell}:=n_{\star} \vee \exp \left(2\left(c_{*}+1\right) /\left(2-c_{*}\right)\right)$, one easily checks that

$z=r+(\log \ell-1) / c_{*} \geq 1$ whenever $r \geq-\frac{1}{2} \log \ell$ and $\ell \geq \hat{\ell}$. If further $1 \leq \ell \leq n / \log n$, then

$$
\rho_{n} \ell+r=c_{*} \ell-\frac{\ell \log n}{c_{*} n}+r \geq m_{\ell}+z,
$$

so (5.3) at $n=\ell$ and such $z$ yields the bound (5.4), possibly with $c \mapsto e c$.

We recall (4.5), (4.9) and take throughout

$$
H_{x}:=[x, x+1] .
$$

The key to this section are the following a-priori barrier estimates adapted from [8, Section 5] (though it is advised to skip the proofs at first reading). 
Lemma 5.3. Let $g_{m}(i):=i \exp \left(c_{*} i+i^{2} / m\right), \beta_{n, k}:=\frac{k}{n} \log n-\log k$ and $z_{h}:=z+h$. For some $c_{1} \geq 1$, all $z \geq 1, k \geq 0, h \in[0, n-k)$ and $i \in \mathbb{Z}_{+}$

$$
\begin{aligned}
& q_{n, k, z}(i ; h):=\mathbb{P}_{s_{n, z}}\left(\min _{j \leq k}\left\{\hat{\eta}_{v}(j)+\psi_{k, h}(j)\right\} \geq 0, \hat{\eta}_{v}(k) \in H_{i-h}\right) \\
& \quad \leq c_{1} 2^{-k}\left(\frac{z_{h}}{\sqrt{k_{n}}} \wedge k\right) e^{\beta_{n, k}} e^{-c_{*} z_{h}} e^{-z_{h}^{2} /(4 m)} g_{m}(i+1), \quad \forall m \in\left[2 k, n^{2}\right]
\end{aligned}
$$

(replacing for $k=0$ the ill-defined factor $\left(\frac{z_{h}}{\sqrt{k_{n}}} \wedge k\right) e^{\beta_{n, k}}$ by 1$)$.

Likewise, for $i, k^{\prime} \in \mathbb{Z}_{+}, n^{\prime}=k^{\prime}+k \in\left(k^{\prime}, n\right), m \in\left[2 k,\left(n-k^{\prime}\right)^{2}\right]$ and $z \geq 0$,

$$
\begin{aligned}
p_{n, k, z}(i) & :=\mathbb{P}\left(\min _{j \in\left(k^{\prime}, n^{\prime}\right]}\left\{\hat{\eta}_{v}(j)\right\} \geq 0, \hat{\eta}_{v}\left(n^{\prime}\right) \in H_{i} \mid \hat{\eta}_{v}\left(k^{\prime}\right)=z\right) \\
& \leq c_{1} \frac{2^{-k} e^{\beta_{n, k}}}{\sqrt{k_{n-k^{\prime}}}}(z \vee 1) e^{-c_{*} z} e^{-(z \vee 1)^{2} /(4 m)} g_{m}(i+1) .
\end{aligned}
$$

The bound (5.8) applies also to $z \in\left[-\rho_{n} k, 0\right]$, now with $m=-4 k$.

Proof. In case $k \geq 1$, setting $a=\rho_{n} n-h$ and $b=\rho_{n}(n-k)-h$, the event considered in (5.6) corresponds to [8, (1.1)] for $L=k, C=1, \varepsilon=\frac{1}{2}-\delta$ and the line $f_{a, b}(j ; k)$ between $(0, a)$ and $(k, b)$, taking there $y=b+i$ and $x=m_{n}+z \geq a$. Having $z \geq 1$ and $m_{n} \geq 1$ yields that $x \geq 2$. Further, with $h \in[0, n-k)$ and $\rho_{n} \geq \rho_{*}$,

$$
y \geq b=\rho_{n}(n-k)-h \geq 1+\frac{1}{10}(n-k)
$$

(the restriction to $y \geq \sqrt{2}$ in [8, (1.1)] clearly can be replaced with $y>1$ there, since for $y \in[1, \sqrt{2})$ one has $\left.H_{y}^{2} / 2 \cap \mathbb{Z} \subset\left(H_{\sqrt{2}}\right)^{2} / 2 \cap \mathbb{Z}\right)$. Here $x / L$ and $y / L$ are not uniformly bounded above but following [8, proof of (4.2)] and utilizing [8, Remark 2.6] to suitably modify [8, (4.16)], we nevertheless arrive at the bound

$$
q_{n, k, z}(i ; h) \leq c \frac{\left(1+z_{h}\right)(1+i)}{k} \sqrt{\frac{x}{k y}} \sup _{w \in H_{y}}\left\{e^{-(x-w)^{2} /(2 k)}\right\}
$$

In addition, whenever $x \geq \sqrt{2}, y, k \geq 1$, we have that

$$
q_{n, k, z}(i ; h) \leq \mathbb{P}_{s_{n, z}}\left(\hat{\eta}_{v}(k) \in H_{i-h}\right) \leq c \sup _{w \in H_{y}}\left\{e^{-(x-w)^{2} /(2 k)}\right\}
$$

(see [9, Lemma 3.6]). Next, since $x \leq c_{*} n+z$, we deduce from (5.9) that

$$
\frac{x}{k y} \leq \frac{20}{k_{n}}\left(c_{*}+\frac{z}{n}\right) \leq \frac{c_{o}}{k_{n}} \exp \left(\frac{z^{2}}{12 n^{2}}\right),
$$

for some constant $c_{o}<\infty$. Further, as $x-a=z_{h}$ we have from (1.1) that

$$
x-b=c_{*} k+z_{h}-\varepsilon_{n, k}, \quad \varepsilon_{n, k}:=\frac{k \log n}{c_{*} n},
$$

and since $\frac{c_{*}^{2}}{2}=\log 2$, we get, for any real $\widetilde{w}$,

$$
\frac{1}{2 k}(x-b-\widetilde{w})^{2}=k \log 2+c_{*}\left(z_{h}-\varepsilon_{n, k}-\widetilde{w}\right)+\frac{1}{2 k}\left(z_{h}-\varepsilon_{n, k}-\widetilde{w}\right)^{2} .
$$


By an elementary inequality, for any $m \geq 2 k$,

$$
\left(z_{h}-\widetilde{w}-\varepsilon_{n, k}\right)^{2} \geq \frac{2 k}{3 m}\left(z_{h}^{2}-3 \widetilde{w}^{2}-3 \varepsilon_{n, k}^{2}\right),
$$

so that by (5.14)

$$
\frac{1}{2 k}(x-b-\widetilde{w})^{2} \geq k \log 2+c_{*}\left(z_{h}-\varepsilon_{n, k}-\widetilde{w}\right)+\frac{z_{h}^{2}}{3 m}-\frac{\widetilde{w}^{2}}{m}-\frac{\varepsilon_{n, k}^{2}}{m} .
$$

With $c_{*} \varepsilon_{n, k}-\log k=\beta_{n, k} \leq 1$ for $k \in[1, n]$ (and $\varepsilon_{n, k}^{2} / k$ uniformly bounded), we plug into the smaller among (5.10) and (5.11) the bounds (5.12) and (5.16) (for $w=b+\widetilde{w}$ and $\left.\widetilde{w} \in H_{i}\right)$, to arrive at (5.7). By definition $q_{n, 0, z}(i ; h)=\mathbf{1}_{z_{h}}(i)$, and since $g_{m}\left(z_{h}+1\right) \geq \exp \left(c_{*} z_{h}+z_{h}^{2} /(4 m)\right)$, clearly (5.7) holds also for $k=0$ (under our convention).

Turning to the proof of (5.8), we consider first $z>0$, proceeding as in the proof of (5.7) with the line $f_{a, b}(j ; k)$ of length $k$ and same slope as in the preceding, now connecting $\left(k^{\prime}, a\right)$ to $\left(n^{\prime}, b\right)$, where $a=\rho_{n}\left(n-k^{\prime}\right)$ and $b=\rho_{n}\left(n-n^{\prime}\right)$. For $x=a+z$ and $y=b+i$ we have $x \geq a>b$ and $y \geq b>0$, thanks to our assumption that $n^{\prime} \in\left(k^{\prime}, n\right)$. By the Markov property of $j \mapsto \eta_{v}(j)$, for such values of $(a, b, x, y)$ the RHS of (5.10) with $h=0$ necessarily bounds the probability $p_{n, k, z}(i)$, and thereafter one merely follows the derivation of (5.7), now with $h=0$ and $n-k^{\prime}$ replacing $n$ when bounding $x / y$. The latter modification results in having $\sqrt{k_{n-k^{\prime}}}$ in (15.8), instead of $\sqrt{k_{n}}$. Next, for $z \leq 0$ we simply lower the barrier line $f_{a, b}(j ; k)$ to start at $a=x=\bar{\varphi}_{n}\left(k^{\prime}\right)+z$, where our assumption that $z \geq-\rho_{n} k$ guarantees having $x \geq \bar{\varphi}_{n}\left(n^{\prime}\right)>0$ (thereby yielding (5.10) ). Here $x /(k y) \leq c_{o} / k_{n-k^{\prime}}$, and $z_{h}=z \leq 0$ allows us to replace the RHS of (5.15) by $\frac{\widetilde{w}^{2}}{2}-\varepsilon_{n, k}^{2}$, yielding the stated form of (5.8).

We conclude this sub-section by adapting the bounds of Lemma 5.3 to the form needed when proving Lemma 4.4 and Lemma 4.2 .

Lemma 5.4. There exists a constant $c_{2}<\infty$ satisfying the following. Fix $\hat{\ell}$ as in Corollary 5.2 and $I_{\ell}$ as in (4.4). For any $z \geq 0, \ell \in\left[\hat{\ell}, \frac{n}{\log n}\right]$ and $k \geq 1$, setting $k^{\prime}=n^{\prime}-k$ with $n^{\prime}$ as in 4.3,

$$
\begin{aligned}
\theta_{n, k, \ell}(z) & :=\mathbb{P}\left(\min _{j \in\left(k^{\prime}, n^{\prime}\right]}\left\{\hat{\eta}_{v}(j)\right\} \geq 0, \hat{\eta}_{v}\left(n^{\prime}\right) \in I_{\ell}, \eta_{\ell}^{\sharp}(v)=0 \mid \hat{\eta}_{v}\left(k^{\prime}\right)=z\right) \\
& \leq c_{2} 2^{-k} e^{\beta_{n, k}}(1 \vee \sqrt{\ell / k})(1+z) e^{-c_{*} z} e^{-z^{2} /(8(k \vee 8 \ell))}
\end{aligned}
$$

If in addition $z \geq 4 h_{\ell}, n \geq 3 \ell$, then for any $r \geq 0$,

$$
\mathbf{p}_{n, k, z}(r):=\mathbb{P}_{s_{n, z}}\left(\min _{j \leq n^{\prime}}\left\{\hat{\eta}_{v}(j)+\psi_{\ell}(j) \mathbf{1}_{j \leq k}\right\}>0, \hat{\eta}_{v}(k) \leq 0, \hat{\eta}_{v}\left(n^{\prime}\right) \in H_{r}\right)
$$

$$
\leq c_{2} 2^{-n^{\prime}} \frac{\psi_{\ell}(k)^{3}}{k_{n^{\prime}}^{3 / 2} \ell^{1 / 2}} e^{-z^{2} /(16 k)} z(r+1) e^{-c_{*}(z-r)} e^{-\frac{r^{2}}{4\left(n^{\prime}-k\right)}} .
$$


Proof. Starting with (5.17), by the Markov property of $\eta_{v}(j)$ at $j=n^{\prime}$ and monotonicity of $y \mapsto \gamma_{n, \ell}(y)$ of (5.4), we have in terms of $p_{n, k, z}(\cdot)$ of (5.8)

$$
\theta_{n, k, \ell}(z) \leq \sum_{r \in I_{\ell}} p_{n, k, z}(r) \gamma_{n, \ell}(r) \text {. }
$$

Plugging the bounds of (5.4) and (5.8) (at $m=2(k \vee 8 \ell)$ ), yields for $c_{1}^{\prime}$ finite

$$
\theta_{n, k, \ell}(z) \leq c_{1}^{\prime} \frac{2^{-k} e^{\beta_{n, k} \sqrt{\ell}}}{\sqrt{k_{n-k^{\prime}}}}(1+z) e^{-c_{*} z} e^{-z^{2} /(8(k \vee 8 \ell))} \sum_{r \in I_{\ell}} \frac{r^{2}}{\ell^{3 / 2}} e^{-r^{2} /(16 \ell)} .
$$

With the latter sum uniformly bounded and $k_{n-k^{\prime}}=k \wedge \ell$, we arrive at (5.17).

Next, turning to establish (5.18), note first that

$$
j_{k^{\prime}}^{\beta} \leq k_{k^{\prime}}^{\beta}+j_{k}^{\beta}, \quad \forall 0 \leq j \leq k<k^{\prime}
$$

when $\beta=1$ and consequently also for all $\beta \in[0,1]$. For $\beta=\delta$, it results with

$$
\psi_{k^{\prime}, h}(j) \leq \psi_{k, h^{\prime}}(j), \quad \text { for } \quad h^{\prime}=\psi_{k^{\prime}, h}(k), \quad j \in[0, k],
$$

with equality at $j=k$. In particular, recalling (4.10) and considering $k^{\prime}=n^{\prime}$, we have that

$$
\psi_{\ell}(j) \leq \psi_{k, h}(j) \quad \text { for } \quad h=\psi_{\ell}(k), \quad j \in[0, k] .
$$

Employing (5.20) to enlarge the event whose probability is $\mathbf{p}_{n, k, z}(i)$, we get by the Markov property of $\eta_{v}(j)$ at $j=k$, in terms of $q_{n, k, z}(\cdot ; \cdot), p_{n, k, z}(\cdot)$ and $h=\psi_{\ell}(k)$, that

$$
\mathbf{p}_{n, k, z}(r) \leq \sum_{i=1}^{h} q_{n, k, z}(h-i ; h) \sup _{z^{\prime} \in H_{-i}}\left\{p_{n, k^{\prime}, z^{\prime}}(r)\right\} .
$$

Substituting first our bound (5.8) at $m=-4 k^{\prime}$, and then (5.7) at $m=2 k$, yields that for some $c_{1}^{\prime}$ finite,

$$
\mathbf{p}_{n, k, z}(r) \leq c_{1} \sum_{i=1}^{h} q_{n, k, z}(h-i ; h) 2^{-k^{\prime}} e^{\beta_{n, k^{\prime}}}\left(k_{k^{\prime}+\ell}^{\prime}\right)^{-1 / 2} e^{c_{*}(r+i)}(r+1) e^{-\frac{r^{2}}{4 k^{\prime}}}
$$

$$
\leq c_{1}^{\prime} h 2^{-n^{\prime}} \frac{e^{\beta_{n, k}+\beta_{n, k^{\prime}}}}{\sqrt{k_{k^{\prime}+\ell}^{\prime} k_{n}}} z_{h} e^{-c_{*} z_{h}} e^{-z^{2} /(8 k)} g_{2 k}(h)(r+1) e^{c_{*} r} e^{-r^{2} /\left(4 k^{\prime}\right)} .
$$

With $\delta \leq \frac{1}{2}$ and $z \geq 4 h_{\ell}$, it follows that

$$
\frac{h^{2}}{2 k} \leq \frac{h_{\ell}^{2}+k_{n^{\prime}}^{2 \delta}}{k} \leq \frac{z^{2}}{16 k}+1 .
$$

Further, recall that $k+k^{\prime}=n^{\prime}$ and $\beta_{n, n^{\prime}} \leq 1$, hence

$$
e^{\beta_{n, k}+\beta_{n, k^{\prime}}}=e^{\beta_{n, n^{\prime}}} \frac{n^{\prime}}{k k^{\prime}} \leq \frac{2 e}{k_{n^{\prime}}} .
$$

Our assumption $n \geq 3 \ell$ results with $k \vee k^{\prime} \geq \ell$ and thereby $k_{k^{\prime}+\ell}^{\prime} k_{n} \geq \ell k_{n^{\prime}}$. Applying the preceding within (5.22), we arrive at (5.18) . 
5.1. Negligible crossings: Proof of Lemma 5.1. Fixing $n, k^{\prime}, h$ as in Lemma 5.1, consider for $u \in V_{k^{\prime}}$, the first time

$$
\tau_{u}:=\min \left\{j \geq 0: \eta_{u}(j) \leq \varphi_{n, k^{\prime}, h}(j)\right\}
$$

that the process $j \mapsto \eta_{u}(j)$ reaches the relevant barrier of (4.8), see Figure 2 , For $z \geq 1$ we have that $\tau_{u} \geq 1$, since $\eta_{u}(0)=\sqrt{2 s_{n, z}}=m_{n}+z>\varphi_{n, k^{\prime}, h}(0)$ under $\mathbb{P}_{s_{n, z}}$. Decomposing $G_{n, k^{\prime}}(h)$ according to the possible values of $\left\{\tau_{u}\right\}$, results with

$$
\mathbb{P}_{s_{n, z}}\left(G_{n, k^{\prime}}(h)\right) \leq \sum_{k=1}^{k^{\prime}} \underbrace{\mathbb{P}_{s_{n, z}}\left(\exists u \in V_{k^{\prime}} \text { such that } \tau_{u}=k\right)}_{\left(\mathrm{I}_{k}\right)} .
$$

The event $\left\{\tau_{u}=k\right\}$ depends only on the value of $u(k) \in V_{k}$. Hence, by the union bound we have that for any fixed $u \in V_{k^{\prime}}$

$$
\left(\mathrm{I}_{k}\right) \leq 2^{k} \mathbb{P}_{s_{n, z}}\left(\tau_{u}=k\right) \text {. }
$$

With $\rho_{n}>1>\delta$, it is easy to verify that $j \mapsto \varphi_{n, k^{\prime}, h}(j)$ is strictly decreasing with $\varphi_{n, k^{\prime}, h}\left(k^{\prime}\right)=\rho_{n}\left(n-k^{\prime}\right)-h \geq 0$. Further, for $b:=\varphi_{n, k^{\prime}, h}(k), \widetilde{b}:=$ $\varphi_{n, k^{\prime}, h}(k+1)$, we get upon conditioning on $\eta_{u}(k)=y$, that

$$
\begin{aligned}
& \mathbb{P}_{s_{n, z}}\left(\tau_{u}=k+1\right) \leq \\
& \quad \sum_{i=0}^{\infty} \mathbb{P}_{s_{n, z}}\left(\tau_{u}>k, \eta_{u}(k) \in H_{b+i}\right) \sup _{y \in H_{b+i}} \mathbb{P}_{\frac{y^{2}}{2}}\left(\eta_{u}(1) \leq \widetilde{b}\right) .
\end{aligned}
$$

Applying [7, Lemma 4.6] at $p=q=1 / 2$ and $\theta=\widetilde{b}^{2} / 2 \leq y^{2} / 2$

$$
\sup _{y \in H_{b+i}} \mathbb{P}_{\frac{y^{2}}{2}}\left(\eta_{u}(1) \leq \widetilde{b}\right) \leq e^{-i^{2} / 4} .
$$

Setting $h^{\prime}=\psi_{k^{\prime}, h}(k)$, we proceed to bound the first probability on the RHS of (5.26). To this end, recall (5.19), yielding that $\varphi_{n, k, h^{\prime}}(j) \leq \varphi_{n, k^{\prime}, h}(j)$ for $j \in[0, k]$, with equality at $j=k$ (see (4.8)-(4.9)). Consequently,

$$
\mathbb{P}_{s_{n, z}}\left(\tau_{u}>k, \eta_{u}(k) \in H_{b+i}\right) \leq q_{n, k, z}\left(i ; h^{\prime}\right)
$$

for $q_{n, k, z}(\cdot ; \cdot)$ of Lemma 5.3. Since $n-k^{\prime} \geq h$ and $\delta<1$, for any $k<k^{\prime}$,

$$
n-k-h^{\prime} \geq k^{\prime}-k-\left(k^{\prime}-k\right)^{\delta}>0,
$$

in which case, by (5.7) we have that for any $i \in \mathbb{Z}_{+}$

$$
q_{n, k, z}\left(i ; h^{\prime}\right) \leq c_{1} 2^{-k} z_{h^{\prime}} e^{-c_{*} z_{h^{\prime}}} e^{-z_{h^{\prime}}^{2} /(8 n)} g_{2 n}(i+1) .
$$

Noting that $\sup _{n \geq 3}\left\{g_{2 n}(i+1)\right\} e^{-i^{2} / 4}$ is summable (and $z_{h^{\prime}} \geq z_{h}$ ), we find upon combining (5.25) $-(5.29)$, that for some $c_{3}$ finite and any $1 \leq k<k^{\prime}$,

$$
\begin{aligned}
\left(\mathrm{I}_{k+1}\right) & \leq 2^{k+1} \sum_{i=0}^{\infty} q_{n, k, z}\left(i ; h^{\prime}\right) e^{-i^{2} / 4} \\
& \leq c_{3}\left(z_{h}+k_{k^{\prime}}^{\delta}\right) e^{-c_{*}\left(z_{h}+k_{k^{\prime}}^{\delta}\right)} e^{-z_{h}^{2} /(8 n)} .
\end{aligned}
$$


Further, with $\varphi_{n, k^{\prime}, h}(1) \leq m_{n}-h$ we have similarly to (5.25) -(5.27) that

$$
\left(\mathrm{I}_{1}\right) \leq 2 \mathbb{P}_{s_{n, z}}\left(\tau_{u}=1\right) \leq 2 \mathbb{P}_{\frac{x^{2}}{2}}\left(\eta_{u}(1) \leq m_{n}-h\right) \leq 2 e^{-z_{h}^{2} / 4}
$$

which is further bounded for $z_{h} \geq z \geq 1$ by the RHS of (5.30) at $k=0$ (possibly increasing the universal constant $c 3$ ). Summing over $k \leq k^{\prime}$ it follows from (5.24) and (5.30) that for some universal $c_{4}<\infty$,

$$
\begin{aligned}
\mathbb{P}_{s_{n, z}}\left(G_{n, k^{\prime}}(h)\right) & \leq c_{3} e^{-c_{*} z_{h}} e^{-z_{h}^{2} /(8 n)} \sum_{k=0}^{k^{\prime}}\left(z_{h}+k_{k^{\prime}}^{\delta}\right) e^{-c_{*} k_{k^{\prime}}^{\delta}} \\
& \leq c_{4} z_{h} e^{-c_{*} z_{h}} e^{-z_{h}^{2} /(8 n)}
\end{aligned}
$$

as claimed in (5.1).

5.2. Comparing barriers: Proof of Lemma 4.2. Hereafter, let $\nu_{n, k, z}(\cdot)$ denote the finite measure on $[0, \infty)$ such that

$$
\nu_{n, k, z}(A):=2^{k} \mathbb{P}_{s_{n, z}}\left(\min _{j \leq k}\left\{\hat{\eta}_{v}(j)\right\}>0, \hat{\eta}_{v}(k) \in A\right),
$$

using the abbreviation $\nu_{n, z}=\nu_{n, n^{\prime}, z}\left(\right.$ where $n^{\prime}=n-\ell$ ). In view of (4.6) and the Markov property of $\left\{\eta_{v}(j)\right\}$ at $j=n^{\prime}$ we have that

$$
\mathbb{E}_{s_{n, z}}\left[\Lambda_{n, \ell}\right]=2^{n^{\prime}} \mathbb{P}_{s_{n, z}}\left(E_{n, \ell}(v)\right)=\int_{I_{\ell}} \gamma_{n, \ell}(y) \nu_{n, z}(d y) .
$$

for $\gamma_{n, \ell}(\cdot)$ of (5.4). Similarly, setting the finite measure on $\left[-h_{\ell}, \infty\right)$

$$
\mu_{n, z}(A):=2^{n^{\prime}} \mathbb{P}_{s_{n, z}}\left(\min _{j \leq n^{\prime}}\left\{\hat{\eta}_{v}(j)+\psi_{\ell}(j)\right\}>0, \hat{\eta}_{v}\left(n^{\prime}\right) \in A\right),
$$

we have by the Markov property and (4.12) that

$$
\mathbb{E}_{s_{n, z}}\left[\Gamma_{n, \ell}\right]=2^{n^{\prime}} \mathbb{P}_{s_{n, z}}\left(F_{n, \ell}(v)\right)=\int_{-h_{\ell}}^{\infty} \gamma_{n, \ell}(y) \mu_{n, z}(d y) .
$$

For $r \geq 0$, recalling $H_{r}=[r, r+1]$, we decompose $\mu_{n, z}\left(H_{r}\right)-\nu_{n, z}\left(H_{r}\right)$ according to the possible values of $\tau:=\max \left\{j<n^{\prime}: \hat{\eta}_{v}(j) \leq 0\right\}$, to arrive at

$$
\mu_{n, z}\left(H_{r}\right)-\nu_{n, z}\left(H_{r}\right) \leq 2^{n^{\prime}} \sum_{k=1}^{n^{\prime}-1} \mathbf{p}_{n, k, z}(r),
$$

for $\mathbf{p}_{n, k, z}(r)$ of Lemma 5.4. By (5.32), (5.34), (5.35) and the monotonicity of $y \mapsto \gamma_{n, \ell}(y)$ we have that

$$
\begin{aligned}
\mathbb{E}_{s_{n, z}}\left[\Gamma_{n, \ell}-\Lambda_{n, \ell}\right] \leq & \sum_{r \notin I_{\ell}} \gamma_{n, \ell}(r) \mu_{n, z}\left(H_{r}\right)+\sum_{r \in I_{\ell}} \gamma_{n, \ell}(r) \sum_{k=1}^{n^{\prime}-1} 2^{n^{\prime}} \mathbf{p}_{n, k, z}(r) \\
& :=\mathrm{I}_{n}(z, \ell)+\mathrm{I}_{n}(z, \ell) .
\end{aligned}
$$

Dealing first with $\mathrm{I}_{n}(z, \ell)$ of (5.36) ), note that $\mu_{n, z}\left(H_{r}\right)=2^{n^{\prime}} q_{n, n^{\prime}}(r+h ; h)$ for $q_{n, k}(i ; h)$ of Lemma 5.3 and $h=h_{\ell}$. Combining (5.4) with (5.7) at $k=n^{\prime}$ 
(where $k_{n}=\ell$ ), and having $z+h_{\ell} \leq 2 z$ (as $z \rightarrow \infty$ before $\ell \rightarrow \infty$ ), yields for some $c_{5}$ finite, any $\ell \geq \hat{\ell}$, large $n$ and all $r \geq-h_{\ell}$

$$
\gamma_{n, \ell}(r) \mu_{n, z}\left(H_{r}\right) \leq c_{5} z e^{-c_{*} z} \frac{\left(r+2 h_{\ell}\right)^{2}}{\ell^{3 / 2}} e^{-r^{2} /(8 \ell)} e^{\left(r+h_{\ell}\right)^{2} / n}
$$

Substituting (5.37) in (5.36) and taking $n \rightarrow \infty$ results with

$$
\varlimsup_{z \rightarrow \infty}\left\{z^{-1} e^{c_{*} z} \varlimsup_{n \rightarrow \infty} \mathrm{I}_{n}(z, \ell)\right\} \leq \varepsilon_{\mathbf{l}}(\ell)
$$

where by our choice (4.4) of $I_{\ell}$, for any $\ell \rightarrow \infty$

$$
\varepsilon_{\mathrm{l}}(\ell):=c_{5} \sum_{r \notin I_{\ell}} \frac{\left(r+2 h_{\ell}\right)^{2}}{\ell^{3 / 2}} e^{-r^{2} /(8 \ell)} \longrightarrow 0 .
$$

In view of (5.4), it suffices to show that for some $\varepsilon_{\| I}(\ell) \rightarrow 0$ and all $r \in I_{\ell}$,

$$
\varlimsup_{z \rightarrow \infty}\left\{z^{-1} e^{c_{*} z} \varlimsup_{n \rightarrow \infty} \sum_{k=1}^{n^{\prime}-1} 2^{n^{\prime}} \mathbf{p}_{n, k, z}(r)\right\} \leq \frac{\varepsilon_{\mathrm{II}}(\ell)}{\sqrt{\ell}}(r+1) e^{c_{*} r},
$$

in order to get the analog of (5.38) for $\mathrm{II}_{n}(z, \ell)$ and thereby complete the proof of the lemma. In view of (5.18) we get (5.39) upon showing that

$$
\lim _{z \rightarrow \infty} \sup _{r \in I_{\ell}} \varlimsup_{n \rightarrow \infty} \sum_{k=1}^{n^{\prime}-1} \psi_{\ell}(k)^{3} k_{n^{\prime}}^{-3 / 2} \exp \left(-\frac{z^{2}}{16 k}-\frac{r^{2}}{4\left(n^{\prime}-k\right)}\right)=0 .
$$

Even without the exponential factor, since $\delta<\frac{1}{6}$ the sum in (5.40) over $\left\{k: k_{n^{\prime}}^{\delta} \geq h_{\ell}\right\}$, where $\psi_{\ell}(k) \leq 2 k_{n^{\prime}}^{\delta}$ is bounded above by

$$
4 \sum_{k \geq h_{\ell}^{1 / \delta}} k^{3 \delta-3 / 2} \leq c h_{\ell}^{\left(3-\frac{1}{2 \delta}\right)} \underset{\ell \rightarrow \infty}{\longrightarrow} 0
$$

Further, the sum in (5.40) over $\left\{k: k_{n^{\prime}}^{\delta}<h_{\ell}\right\}$ has $2 h_{\ell}^{1 / \delta}$ terms, which are uniformly bounded by $\left(2 h_{\ell}\right)^{3} \exp \left(-b_{\ell} /\left(4 h_{\ell}^{1 / \delta}\right)\right)$, where having

$$
b_{\ell}:=\frac{z^{2}}{4} \wedge \inf _{r \in I_{\ell}}\left\{r^{2}\right\}=\frac{\ell}{\log \ell}
$$

makes that sum also negligible, as claimed in (5.40).

5.3. Second moment: proof of Lemma 4.4. In view of (4.7) we have that

$$
\mathbb{E}_{s}\left[\Lambda_{n, \ell}\left(\Lambda_{n, \ell}-1\right)\right]=\sum_{\substack{u, v \in V_{n^{\prime}} \\ u \neq v}} \mathbb{P}_{s}\left(E_{n, \ell}(u) \cap E_{n, \ell}(v)\right)
$$

We recall the definition (4.6) of $E_{n, \ell}(\cdot)$ and split the preceding sum according to the values of $k^{\prime}=|u \wedge v|<n^{\prime}$ and $\hat{\eta}_{v}\left(k^{\prime}\right)>0$. Specifically, having $2^{n^{\prime}+k-1}$ 
such ordered pairs (for $k=n^{\prime}-k^{\prime}$ ), yields the bound

$$
\mathbb{E}_{s_{n, z}}\left[\Lambda_{n, \ell}\left(\Lambda_{n, \ell}-1\right)\right] \leq \sum_{k=1}^{n^{\prime}} 2^{2 k} \int_{0}^{\infty} \theta_{n, k, \ell}^{2}(y) \nu_{n, k^{\prime}, z}(d y)=: \sum_{k=1}^{n^{\prime}} J_{k}
$$

in terms of $\theta_{n, k, \ell}(\cdot)$ and $\nu_{n, k^{\prime}, z}(\cdot)$ of (5.17) and (5.31), respectively. Further, the Markov property of $\eta_{v}(j)$ at $j=k^{\prime}$ yields in terms of $q_{n, k^{\prime}, z}(\cdot ; 0)$ of (5.6)

$$
\mathrm{J}_{\mathrm{k}} \leq \sum_{i=0}^{\infty} 2^{k^{\prime}} q_{n, k^{\prime}, z}(i ; 0)\left[2^{k} \sup _{y \in H_{i}} \theta_{n, k, \ell}(y)\right]^{2} .
$$

Plugging into the preceding the bounds (5.17) and (5.7) (at $m=64 n \leq n^{2}$ ), we find that for some $c_{6}<\infty$, any $k \in\left(0, n^{\prime}\right), z \geq 1$ and $n \geq n_{0}(\ell)$ as in Lemma 5.4 .

$$
\mathrm{J}_{\mathrm{k}} \leq c_{6} e^{\beta_{n, k^{\prime}}+2 \beta_{n, k}}(1 \vee \ell / k) \frac{z}{\sqrt{k_{n}^{\prime}} \wedge k^{\prime}} e^{-c_{*} z} \sum_{i=0}^{\infty}(i+1)^{3} e^{-c_{*} i}
$$

Using (5.23),$\beta_{n, k} \leq 1$ and $k_{n}^{\prime} \geq k_{n^{\prime}}$ we get from (5.44) that for some $c_{7}<\infty$,

$$
\mathrm{J}_{\mathrm{k}} \leq \begin{cases}c_{7} z e^{-c_{*} z} k_{n^{\prime}}^{-3 / 2}, & k_{n^{\prime}} \geq \ell \\ c_{7} e^{-c_{*} z}, & k^{\prime}<\ell \\ c_{7} z e^{-c_{*} z} \sqrt{\ell} k^{-3}, & k<\ell\end{cases}
$$

where for $k<\ell$ we used the alternative bounds $\beta_{n, k} \leq 1-\log k$ and $k_{n}^{\prime} \geq \ell$. Now, (5.45) implies that for all $n$,

$$
z^{-1} e^{c_{*} z} \sum_{k=\ell^{1 / 3}}^{n^{\prime}} \mathrm{J}_{k} \leq c_{7}\left[\sqrt{\ell} \sum_{k=\ell^{1 / 3}}^{\ell-1} k^{-3}+\sum_{k=\ell}^{n^{\prime}-\ell} k_{n^{\prime}}^{-3 / 2}+\sum_{k=n^{\prime}-\ell}^{n^{\prime}} z^{-1}\right] \leq \delta_{\ell, z}
$$

for some $\delta_{\ell, z} \rightarrow 0$, when $z \rightarrow \infty$ followed by $\ell \rightarrow \infty$. Turning to control the remaining sum of $\mathrm{J}_{k}$ over $k<\ell^{1 / 3}$, note that by (5.42), upon comparing (5.4) and (5.17) we find that for some $\varepsilon(\ell) \rightarrow 0$ as $\ell \rightarrow \infty$ and any $n \geq n_{0}(\ell)$,

$$
\sum_{k=1}^{\ell^{1 / 3}} 2^{k} \sup _{y \geq 0}\left\{\theta_{n, k, \ell}(y)\right\} \leq 4^{\ell^{1 / 3}} \sup _{r \in I_{\ell}}\left\{\gamma_{n, \ell}(r)\right\} \leq \varepsilon(\ell) .
$$

To complete the proof of Lemma 4.4, recall (4.6) and (5.31), that for $k^{\prime}=n^{\prime}-k$

$$
2^{k} \int_{0}^{\infty} \theta_{n, k, \ell}(y) \nu_{n, k^{\prime}, z}(d y)=\mathbb{E}_{s_{n, z}}\left[\Lambda_{n, \ell}\right]
$$

Hence, we have on the RHS of (5.43) that

$$
\varlimsup_{z \rightarrow \infty}\left\{z^{-1} e^{c_{*} z} \varlimsup_{n \rightarrow \infty} \sum_{k=1}^{\ell^{1 / 3}} J_{k}\right\} \leq \varepsilon(\ell) \varlimsup_{z \rightarrow \infty}\left\{z^{-1} e^{c_{*} z} \varlimsup_{n \rightarrow \infty} \mathbb{E}_{s_{n, z}}\left[\Lambda_{n, \ell}\right]\right\},
$$

which together with (4.22) and (5.3) imply that the RHS is for all $\ell$ large enough at most $2 c \varepsilon(\ell)$ (i.e. negligible, as claimed). 


\section{The Bessel process: Proof of Proposition 4.3}

Hereafter, set $\lambda_{\ell}(y):=\frac{1}{2}\left(c_{*} \ell+y\right)^{2}, y \geq-c^{*} \ell$, with

$$
\begin{aligned}
& \widehat{\gamma}_{\ell}(y):=\mathbb{P}_{\left[\lambda_{\ell}(y)\right]}\left(\eta_{\ell}^{\sharp}=0, \eta(0) \in c_{*} \ell+I_{\ell}\right), \\
& \left.\widetilde{\gamma}_{\ell}(y):=\mathbb{E}^{\xi}\left\{\mathbb{P}_{\xi\left(\lambda_{\ell}(y)\right)}\left(\eta_{\ell}^{\sharp}=0, \eta(0) \in c_{*} \ell+I_{\ell}\right)\right)\right\},
\end{aligned}
$$

which are $\gamma_{\infty, \ell}(\cdot)$ from (5.4), restricted to $I_{\ell}$ of (4.4), and its regularization by an expectation, denoted $\mathbb{E}^{\xi}$, over the independent Poisson $(\lambda)$ variable $\xi(\lambda)$ at $\lambda=\lambda_{\ell}(y)$. We emphasize that the law of $\xi$ depends on $\ell$ but we suppress this from the notation. We follow this convention of suppressing dependence in $\ell, n$ in many places throughout this section, e.g. in the definitions (6.5), (6.10), (6.17), (6.24) and (6.28) below. Our goal here is to prove Proposition 4.3, with

$$
\alpha_{\ell}:=\frac{1}{\sqrt{\pi \ell}} \int_{0}^{\infty} y e^{c_{*} y} \widetilde{\gamma}_{\ell}(y) d y .
$$

In particular $\alpha_{\ell}<\infty$, since by standard Poisson tail estimates for some $c<\infty$,

$$
\widetilde{\gamma}_{\ell}(y) \leq \mathbb{P}\left(\sqrt{2 \xi\left(\lambda_{\ell}(y)\right)}-c_{*} \ell \in I_{\ell}\right) \leq \exp \left\{-c \operatorname{dist}\left(y, I_{\ell}\right)^{2}\right\}
$$

(see [8, (3.8)]). Omitting hereafter from the notation the (irrelevant) specific choice $v \in V_{n^{\prime}}$, we recall from (4.5), (5.31) and (5.32) that

$$
2^{-n^{\prime}} \mathbb{E}_{s_{n, z}}\left[\Lambda_{n, \ell}\right]=\mathbb{E}_{s_{n, z}}\left(\widehat{\gamma}_{\ell}\left(\eta\left(n^{\prime}\right)-c_{*} \ell\right) ; \min _{j<n^{\prime}}\{\hat{\eta}(j)\}>0\right) .
$$

The first step towards Proposition 4.3 is our next lemma, utilizing the Markov structure from [8, Lemma 3.1] to estimate the barrier probabilities on the RHS of (6.4) via the law $\mathbb{P}_{x}^{Y}$ of a 0 -dimensional Bessel process $\left\{Y_{t}\right\}$, starting at $Y_{1}=x$. To this end, define for $\kappa \in \mathbb{R}$ the events

$$
B_{\kappa}:=\bigcap_{j=1}^{n^{\prime}}\left\{Y_{j}>\bar{\varphi}_{n}(j)+\kappa \psi_{\ell}(j)\right\},
$$

in terms of the barrier notations (4.3), (4.9), (4.10), and associate to each $[0,1]$-valued $g(\cdot)$, the function

$$
\widetilde{g}(w):=\mathbb{E}^{\xi}\left[g\left(\sqrt{2 \xi\left(w^{2} / 2\right)}\right)\right],
$$

so in particular $g(w)=\widehat{\gamma}_{\ell}\left(w-c_{*} \ell\right)$ yields $\widetilde{g}(w)=\widetilde{\gamma}_{\ell}\left(w-c_{*} \ell\right)$.

Lemma 6.1. There exist $U_{s} \stackrel{\text { dist }}{\longrightarrow} U_{\infty}$, a centered Gaussian of variance $1 / 2$, and $\varepsilon_{\ell} \rightarrow 0$ as $\ell \rightarrow \infty$, such that for $s=s_{n, z}$, any $[0,1]$-valued $g(\cdot)$ supported on $\left[\bar{\varphi}_{n}\left(n^{\prime}\right), \infty\right)$ and $z \geq \ell$,

$$
\begin{aligned}
\left(1-\varepsilon_{\ell}\right)^{-1} \mathbb{E}^{Y, s}\left(\widetilde{g}\left(Y_{n^{\prime}}\right) ; B_{-1}\right) & \geq \mathbb{E}_{s}\left(g\left(\eta\left(n^{\prime}\right)\right) ; \min _{j<n^{\prime}}\{\widehat{\eta}(j)\}>0\right) \\
& \geq\left(1-\varepsilon_{\ell}\right) \mathbb{E}^{Y, s}\left(\widetilde{g}\left(Y_{n^{\prime}}\right) ; B_{2}\right),
\end{aligned}
$$


where $\mathbb{E}^{Y, s}$ denotes expectation with respect to a 0 -dimensional Bessel process starting at $Y_{1}=U_{s}+\sqrt{2 s}$. Further, for some $\delta>0$,

$$
\sup _{s}\left\{\mathbb{E}\left[e^{\delta U_{s}^{2}}\right]\right\}<\infty \text {. }
$$

Proof. Recall from [8, Lemma 3.1] the time in-homogeneous Markov chain

$$
\left(\eta(0)=\sqrt{2 s}, Y_{1}, \eta(1), \cdots, Y_{n^{\prime}}, \eta\left(n^{\prime}\right), \cdots\right),
$$

of law $\mathbb{Q}_{1}^{s}$. From [8, Lemma 3.1(a)] we have that $\mathbb{Q}\left[g(\eta(j)) \mid Y_{j}\right]=\widetilde{g}\left(Y_{j}\right)$ of (6.6), and that $Y_{1}=\sqrt{2 \mathcal{L}_{1}(s)}$ for a $\Gamma(s, 1)$-random variable $\mathcal{L}_{1}(s)$. Set $U_{s}:=$ $\sqrt{2 \mathcal{L}_{1}(s)}-\sqrt{2 s}$ and note that by [8, Lemma $\left.3.1(\mathrm{~d}, \mathrm{e})\right]$, the random variables $\{\eta(j), j \geq 0\}$ and $\left\{Y_{j}, j \geq 1\right\}$ have respectively, the marginal laws $\mathbb{P}_{s}$ and $\mathbb{P}^{Y, s}$.

Standard large deviations for Gamma variables yield (6.9) with $\delta<1 / 2$ (c.f. [8, (3.13)]). Recall that $\left(\mathcal{L}_{1}(s)-s\right) / \sqrt{2 s} \stackrel{\text { dist }}{\Longrightarrow} U_{\infty}$ when $s \rightarrow \infty$ (by the CLT $)$, hence the same convergence applies for $U_{s}=f_{s}\left(\left(\mathcal{L}_{1}(s)-s\right) / \sqrt{2 s}\right)$ and $f_{s}(\cdot)$ of (2.16). In addition, setting for $k \in \mathbb{N}$ the events

$$
A_{k}:=\bigcap_{j=0}^{k}\left\{\eta(j)>\bar{\varphi}_{n}(j)\right\}
$$

we have by the preceding and (6.5) that the bound (6.7) follows from

$$
\mathbb{Q}_{1}^{s}\left(g\left(\eta\left(n^{\prime}\right)\right) ; A_{n^{\prime}} \cap B_{-1}\right) \geq\left(1-\varepsilon_{\ell}\right) \mathbb{E}_{s}\left(g\left(\eta\left(n^{\prime}\right)\right) ; A_{n^{\prime}}\right)
$$

(taking $k=n^{\prime}$ due to the assumed support of $g(\cdot)$ and including $j=0$ at no loss of generality since $z>0$ ). Now, recall from [8, Lemma 3.1(b)] that

$$
\mathbb{Q}_{1}^{s}\left(g\left(\eta\left(n^{\prime}\right)\right) ; A_{n^{\prime}} \cap B_{-1}\right)=\mathbb{E}_{s}\left(g\left(\eta\left(n^{\prime}\right)\right) \prod_{j=1}^{n^{\prime}} F_{j}^{\eta} ; A_{n^{\prime}}\right),
$$

where if

$$
\sqrt{\eta^{2}(j-1) / 2+\eta^{2}(j) / 2}>\bar{\varphi}_{n}(j)
$$

then by [8, (3.14)],

$$
F_{j}^{\eta}:=\mathbb{Q}_{1}^{s}\left(Y_{j}>\bar{\varphi}_{n}(j)-\psi_{\ell}(j) \mid \eta(j-1), \eta(j)\right) \geq 1-c e^{-c \psi_{\ell}^{2}(j)} .
$$

Since (6.13) holds on the event $A_{n^{\prime}}$ for $j=1, \ldots, n^{\prime}$ recalling (4.10) that $\psi_{\ell}(j)=\psi_{\ell}\left(n^{\prime}-j\right)$ and splitting the product on the LHS of (6.12) to $j>n / 2$ and $j \leq n / 2$, yields the inequality (6.11), and thereby (6.7), with

$$
\varepsilon_{\ell}=1-\prod_{j=0}^{\infty}\left[1-c e^{-c\left(j^{\delta}+h_{\ell}\right)^{2}}\right]^{2},
$$

which converge to zero when $\ell \rightarrow \infty$.

Recall from [8, (3.4)] the notation $\mathbb{Q}_{2}^{x^{2} / 2}$ for the law of the Markov chain $\left(Y_{1}, \eta(1), \cdots\right)$ started at $Y_{1}=x$. To see (6.8), we will show

$$
\mathbb{Q}_{2}^{x^{2} / 2}\left(\widetilde{g}\left(Y_{n^{\prime}}\right) ; A_{n^{\prime}-1} \cap B_{2}\right) \geq\left(1-\varepsilon_{\ell}\right) \mathbb{P}_{x}^{Y}\left(\widetilde{g}\left(Y_{n^{\prime}}\right) ; B_{2}\right) .
$$


Taking the expectation over $x$ with respect to the law of $Y_{1}$ under $Q_{1}^{s}$ and arguing as in the proof of (6.7) will then give (6.8). Turning to establishing (6.15), recall from [8, Lemma 3.1(c)] that

$$
\mathbb{Q}_{2}^{x^{2} / 2}\left(\widetilde{g}\left(Y_{n^{\prime}}\right) ; A_{n^{\prime}-1} \cap B_{2}\right)=\mathbb{P}_{x}^{Y}\left(\widetilde{g}\left(Y_{n^{\prime}}\right) \prod_{j=1}^{n^{\prime}-1} F_{j}^{Y} ; B_{2}\right),
$$

where by $[\underline{8},(3.16)]$,

$$
F_{j}^{Y}:=\mathbb{Q}_{2}^{x^{2} / 2}\left(\eta(j)>\bar{\varphi}_{n}(j) \mid Y_{j}, Y_{j+1}\right) \geq 1-c e^{-c \psi_{\ell}^{2}(j)},
$$

provided that

$$
\sqrt{Y_{j} Y_{j+1}} \geq \bar{\varphi}_{n}(j)+\psi_{\ell}(j)
$$

On $B_{2}$, we have that for any $j<n^{\prime}$, and all $\ell$ larger than some fixed universal constant,

$$
\sqrt{Y_{j} Y_{j+1}}>\bar{\varphi}_{n}(j+1)+2 \psi_{\ell}(j+1) \geq \bar{\varphi}_{n}(j)+\psi_{\ell}(j) .
$$

In particular, with (6.16) holding on $B_{2}$ for any $j \in\left\{1, \ldots, n^{\prime}-1\right\}$, by the same reasoning as before, this yields the inequality (6.15), and hence also (6.8).

We next estimate the barrier probabilities for $\left\{Y_{j}\right\}$ in terms of the law $\mathbb{P}_{x}^{W}$ of a Brownian motion $\left\{W_{t}\right\}$, starting at $W_{1}=Y_{1}=x$. For $0 \leq T<T^{\prime} \leq n^{\prime}$, introduce the events

$$
D_{ \pm 2 \psi, T, T^{\prime}}:=\left\{W_{t}>\bar{\varphi}_{n}(t) \pm 2 \psi_{\ell}(t), \forall t \in\left[T+1, T^{\prime}\right]\right\},
$$

using hereafter $D_{\kappa h_{\ell}, T, T^{\prime}}$ if $\pm 2 \psi_{\ell}(t)$ in (6.17) is replaced by the constant function $\kappa h_{\ell}$, with abbreviated notation $D_{ \pm 2 \psi, T}$ when $T^{\prime}=n^{\prime}-T$ and $D_{ \pm 2 \psi}$ for $D_{ \pm 2 \psi, 0}$. See Figure 3 for a pictorial description. Recall the sets $B_{\kappa}$, see (6.5).

Lemma 6.2. For $\widehat{g}(w):=\widetilde{g}(w) / \sqrt{w}$, some $\varepsilon_{\ell} \rightarrow 0$, any $\widetilde{g}(\cdot)$, n, $\ell$ as in Lemma 6.1 and all $x>0$,

$$
\begin{aligned}
\mathbb{E}_{x}^{Y}\left(\widetilde{g}\left(Y_{n^{\prime}}\right) ; B_{2}\right) & \geq\left(1-\varepsilon_{\ell}\right) \sqrt{x} \mathbb{E}_{x}^{W}\left(\widehat{g}\left(W_{n^{\prime}}\right) ; D_{2 \psi}\right), \\
\mathbb{E}_{x}^{Y}\left(\widetilde{g}\left(Y_{n^{\prime}}\right) ; B_{-1}\right) & \leq\left(1-\varepsilon_{\ell}\right)^{-1} \sqrt{x} \mathbb{E}_{x}^{W}\left(\widehat{g}\left(W_{n^{\prime}}\right) ; D_{-2 \psi}\right) .
\end{aligned}
$$

Proof. Recall that up to the absorption time $\tau_{*}:=\inf \left\{t>1: Y_{t}=0\right\}$, the 0-dimensional Bessel process satisfies the SDE

$$
Y_{t}=W_{t}-\int_{1}^{t} \frac{1}{2 Y_{s}} d s, \quad Y_{1}=W_{1}=x,
$$

with $\left\{W_{t}\right\}$ having the Brownian law $\mathbb{P}_{x}^{W}$. Further, the event $\left\{Y_{n^{\prime}}>0\right\}$ implies that $\left\{\tau_{*}>n^{\prime}\right\}$, in which case by Girsanov's theorem and monotone convergence, we have that for any bounded $\mathcal{F}_{n^{\prime}}$-measurable $Z$,

$$
\mathbb{E}_{x}^{Y}\left(Z ; Y_{n^{\prime}}>0\right)=\mathbb{E}_{x}^{W}\left(Z \sqrt{\frac{x}{W_{n^{\prime}}}} e^{-\frac{3}{8} \int_{1}^{n^{\prime}}\left(W_{s}\right)^{-2} d s} ; \inf _{t \in\left[1, n^{\prime}\right]}\left\{W_{t}\right\}>0\right) .
$$




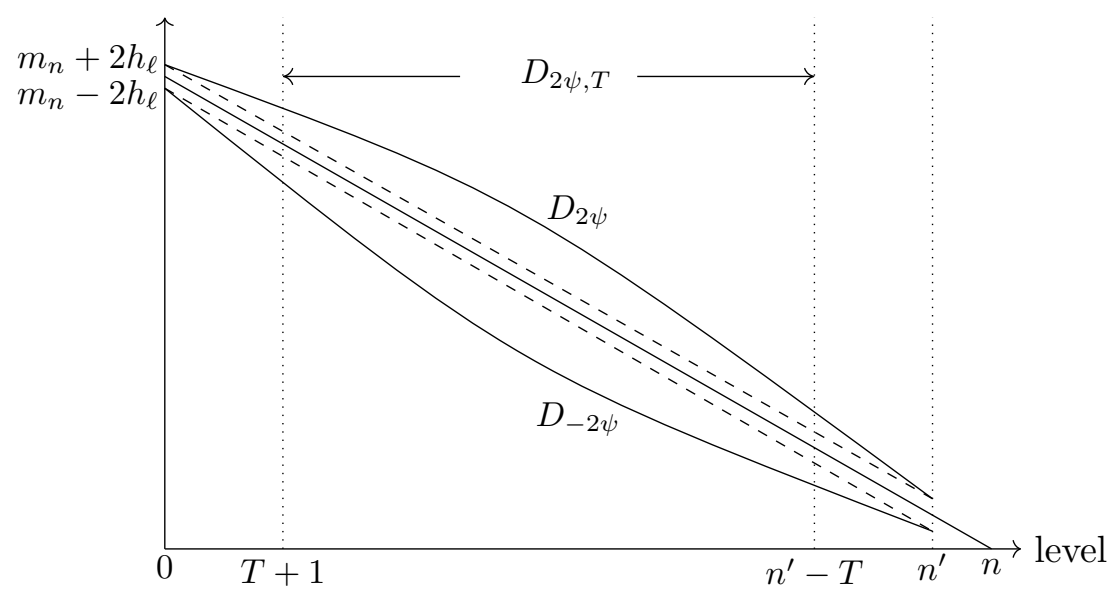

Figure 3. The curves in the events $D_{ \pm 2 h_{\ell}}$ (dashed lines) and $D_{ \pm 2 \psi}$ (curved, solid lines). The event $D_{2 \psi, T}$ involves the curves between $T+1$ and $n^{\prime}-T$.

With $B_{2}$ containing the event corresponding to $D_{2 \psi}$ for the process $Y_{t}$, we get (6.18) by considering (6.20) for $Z=\widetilde{g}\left(W_{n^{\prime}}\right) \mathbf{1}_{D_{2 \psi}}$. Indeed, the event $D_{2 \psi}$ implies that $\inf _{t \leq n^{\prime}}\left\{W_{t}-\bar{\varphi}_{n}(t)\right\} \geq 0$, hence

$$
e^{-\frac{3}{8} \int_{1}^{n^{\prime}}\left(W_{s}\right)^{-2} d s} \geq e^{-\frac{3}{8} \int_{1}^{n^{\prime}}(n-s)^{-2} d s} \geq e^{-\frac{3}{8 \ell}}:=1-\varepsilon_{\ell}
$$

with $\varepsilon_{\ell} \rightarrow 0$ as $\ell \rightarrow \infty$. Next, for all $\ell$ larger than some universal constant,

$$
\inf _{t \in\left[1, n^{\prime}\right]}\left\{\bar{\varphi}_{n}(t)-2 \psi_{\ell}(t)\right\}=\bar{\varphi}_{n}\left(n^{\prime}\right)-2 h_{\ell}>0,
$$

and with $B^{\prime}:=\left\{W_{j}>\bar{\varphi}_{n}(j)-\psi_{\ell}(j), j=1,2, \ldots, n^{\prime}\right\}$, it suffices for (6.19) to show that

$$
\mathbb{E}_{x}^{W}\left(\widehat{g}\left(W_{n^{\prime}}\right) ; D_{-2 \psi} \cap B^{\prime}\right) \geq\left(1-\varepsilon_{\ell}\right) \mathbb{E}_{x}^{W}\left(\widehat{g}\left(W_{n^{\prime}}\right) ; B^{\prime}\right) .
$$

To this end, since $\phi_{t}:=\bar{\varphi}_{n}(t)-2 \psi_{\ell}(t)$ is a convex function, we get upon conditioning on $\left\{W_{1}, W_{2}, \ldots, W_{n^{\prime}}\right\}$, that

$$
\mathbb{E}_{x}^{W}\left(\widehat{g}\left(W_{n^{\prime}}\right) ; D_{-2 \psi} \cap B^{\prime}\right) \geq \mathbb{E}_{x}^{W}\left(\widehat{g}\left(W_{n^{\prime}}\right) \prod_{j=1}^{n^{\prime}-1} F_{j}^{W} ; B^{\prime}\right),
$$

where by the reflection principle (see [8, (2.1)] or [12, Lemma 2.2]),

$$
\begin{aligned}
F_{j}^{W} & :=\mathbb{P}^{W}\left(\min _{u \in[0,1]}\left\{W_{j+u}-f_{\phi_{j}, \phi_{j+1}}(u ; 1)\right\}>0 \mid W_{j}, W_{j+1}\right) \\
& =1-\exp \left(-2\left(W_{j}-\phi_{j}\right)\left(W_{j+1}-\phi_{j+1}\right)\right),
\end{aligned}
$$

with $f_{a, b}(\cdot ; 1)$ denoting the line segment between $(0, a)$ and $(1, b)$. On the event $B^{\prime}$ we thus have that $F_{j}^{W} \geq 1-\exp \left(-2 \psi_{\ell}(j) \psi_{\ell}(j+1)\right)$ for all $j \in$ 
$\left\{1,2, \ldots, n^{\prime}-1\right\}$, thereby in analogy with (6.14), establishing (6.21) for

$$
\varepsilon_{\ell}=1-\prod_{j=0}^{\infty}\left[1-e^{-2\left(j^{\delta}+h_{\ell}\right)\left((j+1)^{\delta}+h_{\ell}\right)}\right]^{2},
$$

which converges to zero as $\ell \rightarrow \infty$.

6.1. Proof of Proposition 4.3, Taking $s=s_{n, z}$ yields that $W_{1}=m_{n}+z+$ $U_{s}$. For such $W_{1}$ let

$$
\alpha_{n, \ell, z}^{( \pm)}:=z^{-1} e^{c_{*} z} 2^{n^{\prime}} \mathbb{E}\left[\sqrt{W_{1} / W_{n^{\prime}}} \widetilde{\gamma}_{\ell}\left(W_{n^{\prime}}-c_{*} \ell\right) \mathbf{q}_{\tilde{n}}^{( \pm)}\left(W_{1}, W_{n^{\prime}}\right)\right],
$$

with $\widetilde{n}:=n^{\prime}-1$ denoting our barrier length and $\mathbf{q}_{\widetilde{n}}^{( \pm)}(x, w):=\mathbf{q}_{\widetilde{n}, 0}^{( \pm)}(x, w)$ for the corresponding non-crossing probabilities

$$
\mathbf{q}_{\tilde{n}, T}^{( \pm)}(x, w):=\mathbb{P}_{x}^{W}\left(D_{ \pm 2 \psi, T} \mid W_{n^{\prime}}=w\right) .
$$

Combining (6.4) with Lemmas 6.1 and 6.2 for $g(\cdot)=\widehat{\gamma}_{\ell}\left(\cdot-c_{*} \ell\right)$ and $\widetilde{g}(\cdot)=$ $\widetilde{\gamma}_{\ell}\left(\cdot-c_{*} \ell\right)$, respectively, we have that

$$
\left(1-\varepsilon_{\ell}\right)^{-2} \alpha_{n, \ell, z}^{(-)} \geq z^{-1} e^{c_{*} z} \mathbb{E}_{s_{n, z}}\left[\Lambda_{n, \ell}\right] \geq\left(1-\varepsilon_{\ell}\right)^{2} \alpha_{n, \ell, z}^{(+)} .
$$

The proof of Proposition 4.3 thus amounts to showing that for any $\epsilon>0$ and all large enough $\ell$,

$$
(1+\epsilon)^{3} \alpha_{\ell} \geq \varlimsup_{z \rightarrow \infty} \varlimsup_{n \rightarrow \infty}\left\{\alpha_{n, \ell, z}^{(-)}\right\} \geq \varliminf_{z \rightarrow \infty} \varliminf_{n \rightarrow \infty}\left\{\alpha_{n, \ell, z}^{(+)}\right\} \geq(1-\epsilon)^{3} \alpha_{\ell} .
$$

To this end, setting $z^{\prime}=z+U_{s}$ and $W_{n^{\prime}}=c_{*} \ell+y$, we write (6.23) explicitly as

$\alpha_{n, \ell, z}^{( \pm)}=\frac{e^{c_{*} z} 2^{n^{\prime}}}{z \sqrt{2 \pi \tilde{n}}} \mathbb{E}\left[\int \widetilde{\gamma}_{\ell}(y) d y \frac{\sqrt{m_{n}+z^{\prime}}}{\sqrt{c_{*} \ell+y}} \mathrm{q}_{\widetilde{n}}^{( \pm)}\left(m_{n}+z^{\prime}, c_{*} \ell+y\right) e^{-\left(m_{n}-c_{*} \ell+z^{\prime}-y\right)^{2} / 2 \widetilde{n}}\right]$,

with the expectation over $z^{\prime}$. Hereafter $\ell \leq n / \log n$ so $\left|c_{*}-\rho_{n}\right| \ell \leq 1$ and $D_{ \pm 2 \psi}$ imposes heights $a^{( \pm)}=\rho_{n} \widetilde{n}+b^{( \pm)}, b^{( \pm)}=c_{*} \ell \pm 2 h_{\ell}$ at barrier end points. Thus, in the preceding formula one needs only consider $z^{\prime}, y \geq \pm 2 h_{\ell}$. Recall (5.13). With $m_{n}-c_{*} \ell=c_{*} n^{\prime}-\varepsilon_{n, n}$, upon setting $\Delta_{n}:=\frac{1}{2 \widetilde{n}}\left(z^{\prime}-y+c_{*}-\varepsilon_{n, n}\right)^{2}$, we then get similarly to (5.14) that

$$
\frac{1}{2 \widetilde{n}}\left(c_{*} n^{\prime}+z^{\prime}-y-\varepsilon_{n, n}\right)^{2}=\left(n^{\prime}+1\right) \log 2+c_{*}\left(z^{\prime}-y-\varepsilon_{n, n}\right)+\Delta_{n} .
$$

Since $c_{*} \varepsilon_{n, n}=\log n$, this simplifies our formula for $\alpha_{n, \ell, z}^{( \pm)}$to

$$
\begin{aligned}
\alpha_{n, \ell, z}^{( \pm)} & =\frac{1}{\sqrt{\pi \ell}} \int_{ \pm 2 h_{\ell}}^{\infty} e^{c_{*} y} \widetilde{\gamma}_{\ell}(y) \frac{\widehat{f}_{n, \ell, z}^{( \pm)}(y)}{\sqrt{1+y /\left(c_{*} \ell\right)}} d y \\
\widehat{f}_{n, \ell, z}^{( \pm)}(y) & :=\frac{n}{2 \sqrt{2} z} \mathbb{E}\left[\frac{\sqrt{m_{n}+z^{\prime}}}{\sqrt{c_{*} \widetilde{n}}} \mathbf{q}_{\widetilde{n}}^{( \pm)}\left(m_{n}+z^{\prime}, c_{*} \ell+y\right) e^{-c_{*} U_{s}} e^{-\Delta_{n}}\right] .
\end{aligned}
$$

By our uniform tail estimate (6.9) for $U_{s}$ and the tail bound (6.3) on $\widetilde{\gamma}_{\ell}(y)$, up to an error $\varepsilon_{n} \rightarrow 0$ as $n \rightarrow \infty$, we can restrict the evaluation of $\widehat{f}_{n, \ell, z}^{( \pm)}(y)$ 
to $\left|z^{\prime}\right|+y \leq C \sqrt{\log n}$. This forces $m_{n}+z^{\prime}=c_{*} \widetilde{n}\left(1+\varepsilon_{n}\right)$ and eliminates $\Delta_{n}$, thereby allowing us to replace $\hat{f}_{n, \ell, z}^{( \pm)}(y)$ in (6.26) by

$$
f_{n, \ell, z}^{( \pm)}(y)=\mathbb{E}\left[\frac{e^{-c_{*} U_{s}}}{\sqrt{2} z} \frac{\widetilde{n}}{2} \mathbf{q}_{\tilde{n}}^{( \pm)}\left(m_{n}+z^{\prime}, c_{*} \ell+y\right)\right] .
$$

Recalling the events $D_{\kappa h_{\ell}, T}$, see below (6.17), we further consider the barrier probabilities

$$
\widetilde{\mathbf{q}}_{\tilde{n}, T}^{( \pm)}(x, w):=\mathbb{P}_{x}^{W}\left(D_{ \pm 2 h_{\ell}, T} \mid W_{n^{\prime}}=w\right),
$$

using the abbreviated notation $\widetilde{\mathrm{q}}_{\tilde{n}}^{( \pm)}(x, w)=\widetilde{\mathrm{q}}_{\tilde{n}, 0}^{( \pm)}(x, w)$. Let $\mathbb{P}_{x \rightarrow w}^{\left[t_{1}, t_{2}\right]}\left(A_{m(t)}\right)$ denote the probability that the Brownian bridge, taking the value $x$ at $t_{1}$ and $w$ at $t_{2}$ remains above the barrier $m(t)$ on the interval $\left[t_{1}, t_{2}\right]$. Recall from [12, Lemma 2.2] that for a linear barrier $m(t)$,

$$
\mathbb{P}_{x \rightarrow w}^{\left[t_{1}, t_{2}\right]}\left(A_{m(t)}\right)=1-e^{-2\left(x-m\left(t_{1}\right)\right)_{+}\left(w-m\left(t_{2}\right)\right)_{+} /\left(t_{2}-t_{1}\right)} .
$$

It follows that

$$
\widetilde{\mathbf{q}}_{\tilde{n}}^{( \pm)}(x, w)=\mathbb{P}_{x \rightarrow w}^{\left[1, n^{\prime}\right]}\left(A_{\bar{\varphi}_{n}(t) \pm 2 h_{\ell}}\right)=1-e^{-2\left(x-a^{( \pm)}\right)_{+}\left(w-b^{( \pm)}\right)_{+} / \tilde{n}},
$$

yielding for $x-a^{( \pm)}=z^{\prime} \mp 2 h_{\ell}$ and $w-b^{( \pm)}=y \mp 2 h_{\ell}$ which are both $O(\sqrt{\log n})$,

$$
\widetilde{\mathrm{q}}_{\widetilde{n}}^{( \pm)}\left(m_{n}+z^{\prime}, c_{*} \ell+y\right)=\frac{2+\varepsilon_{n}}{\widetilde{n}}\left(z^{\prime} \mp 2 h_{\ell}\right)\left(y \mp 2 h_{\ell}\right) .
$$

Note further that

$$
\widetilde{\mathrm{q}}_{\widetilde{n}, T}^{( \pm)}(x, w)=\mathbb{E}_{x}^{W}\left[\mathbb{P}_{W_{T+1} \rightarrow W_{n^{\prime}-T}}^{\left[T+1, n^{\prime}-T\right]}\left(A_{\bar{\varphi}_{n}(t) \pm 2 h_{\ell}}\right) \mid W_{n^{\prime}}=w\right] .
$$

The next lemma paraphrases [12, Proposition 6.1] (with the proof given there also yielding the claimed uniformity).

Lemma 6.3. For each $\epsilon>0$ there exist $T_{\epsilon}, n_{\epsilon}$ finite so that, for any $\ell \geq 0$, $T \in\left[T_{\epsilon}, \frac{1}{2} \widetilde{n}\right], x-a^{( \pm)}, w-b^{( \pm)} \in[0, \log \widetilde{n}]$ and all $\widetilde{n}>n_{\epsilon}$

$$
(1-\epsilon) \widetilde{\mathbf{q}}_{\tilde{n}, T}^{(+)}(x, w) \leq \mathbf{q}_{\tilde{n}, T}^{(+)}(x, w) \leq \mathbf{q}_{\tilde{n}, T}^{(-)}(x, w) \leq(1+\epsilon) \widetilde{\mathrm{q}}_{\tilde{n}, T}^{(-)}(x, w) .
$$

Fixing $\epsilon>0$, we bound separately $f_{n, \ell, z}^{( \pm)}(y)$. Starting with $f_{n, \ell, z}^{(-)}(y)$, we have from (6.27), using the fact that $\mathrm{q}_{\widetilde{n}}^{(-)} \leq \mathrm{q}_{\widetilde{n}, T_{\epsilon}}^{(-)}$and the RHS of (6.33), that

$$
\begin{aligned}
f_{n, \ell, z}^{(-)}(y) & \leq \mathbb{E}\left[\frac{e^{-c_{*} U_{s}}}{\sqrt{2} z} \frac{\widetilde{n}}{2} \mathrm{q}_{\widetilde{n}, T_{\epsilon}}^{(-)}\left(m_{n}+z^{\prime}, c_{*} \ell+y\right)\right] \\
& \leq(1+\epsilon) \mathbb{E}\left[\frac{e^{-c_{*} U_{s}}}{\sqrt{2} z} \frac{\widetilde{n}}{2} \widetilde{\mathrm{q}}_{\tilde{n}, T_{\epsilon}}^{(-)}\left(m_{n}+z^{\prime}, c_{*} \ell+y\right)\right] .
\end{aligned}
$$

Turning to evaluate $\widetilde{\mathbf{q}}_{\tilde{n}, T}^{( \pm)}\left(m_{n}+z^{\prime}, c_{*} \ell+y\right)$, we get from (6.32) and (6.29) that

$$
\widetilde{\mathbf{q}}_{\tilde{n}, T}^{( \pm)}\left(m_{n}+z^{\prime}, c_{*} \ell+y\right) \leq \frac{2}{\widetilde{n}-2 T} \mathbb{E}\left[\left(\bar{Z} \mp 2 h_{\ell}\right)_{+}\left(\bar{Y} \mp 2 h_{\ell}\right)_{+}\right],
$$


where $(\bar{Z}, \bar{Y})$ follow the joint Gaussian distribution of

$$
\left(W_{T+1}-\bar{\varphi}_{n}(T+1), W_{n^{\prime}-T}-\bar{\varphi}_{n}\left(n^{\prime}-T\right)\right)
$$

given $W_{1}=m_{n}+z^{\prime}$ and $W_{n^{\prime}}=c_{*} \ell+y$. It is further easy to verify that

$$
\operatorname{Cov}(\bar{Z}, \bar{Y})=T\left[\begin{array}{ll}
1 & 0 \\
0 & 1
\end{array}\right]+\frac{T^{2}}{\widetilde{n}}\left[\begin{array}{cc}
-1 & 1 \\
1 & -1
\end{array}\right]
$$

and that

$$
\mathbb{E}[(\bar{Z}, \bar{Y})]-\left[z^{\prime}+c_{*}+\frac{T}{\widetilde{n}}\left(y-z^{\prime}\right), y+\frac{T}{\widetilde{n}}\left(z^{\prime}-y\right)\right]=o_{\widetilde{n}}(1)
$$

independently of $\left(z^{\prime}, y\right)$, decaying to zero when $\widetilde{n} \rightarrow \infty$ with $\ell, T$ kept fixed. From this we get, in view of (6.34) and (6.35), that

$$
\varlimsup_{z \rightarrow \infty} \varlimsup_{n \rightarrow \infty}\left\{f_{n, \ell, z}^{(-)}(y)\right\} \leq(1+\epsilon)\left(y+3 h_{\ell}\right) \lim _{z, s \rightarrow \infty} \mathbb{E}\left[\frac{\left(z^{\prime}+3 h_{\ell}\right)_{+}}{\sqrt{2} z} e^{-c_{*} U_{s}}\right]
$$

provided $h_{\ell} \geq c_{*}+\sqrt{T_{\epsilon} /(2 \pi)}$. In addition, with $\mathcal{B}=\left\{\left|U_{s}\right|<z / 2\right\}$, or without such restriction, we get thanks to (6.9), via dominated convergence that

$$
\lim _{z, s \rightarrow \infty} \mathbb{E}\left[\mathbf{1}_{\mathcal{B}} \frac{\left(z^{\prime} \pm 3 h_{\ell}\right)_{+}}{\sqrt{2} z} e^{-c_{*} U_{s}}\right]=\frac{1}{\sqrt{2}} \mathbb{E}\left(e^{-c_{*} U_{\infty}}\right)=\frac{1}{\sqrt{2}} e^{c_{*}^{2} / 4}=1 .
$$

(Recall Lemma 6.1 that $U_{\infty} \sim N(0,1 / 2)$.) Combined with the previous display, we obtain

$$
\varlimsup_{z \rightarrow \infty} \varlimsup_{n \rightarrow \infty}\left\{f_{n, \ell, z}^{(-)}(y)\right\} \leq(1+\epsilon)\left(y+3 h_{\ell}\right) .
$$

Note that for $\ell \rightarrow \infty$

$$
\frac{1}{\sqrt{\pi \ell}} \int_{-2 h_{\ell}}^{\infty} e^{c_{*} y} \widetilde{\gamma}_{\ell}(y) \frac{y+3 h_{\ell}}{\sqrt{1+y /\left(c_{*} \ell\right)}} d y \leq \alpha_{\ell}(1+\epsilon) .
$$

(Due to (6.3) the contribution to $\alpha_{\ell}$ outside $\left[\sqrt{\ell} /\left(2 r_{\ell}\right), 2 r_{\ell} \sqrt{\ell}\right]$ is negligible, whereas within that interval $y / \ell \rightarrow 0$ and $h_{\ell} / y \rightarrow 0$.) Combining (6.26), (6.38) and (6.39) yields the LHS of (6.25), thereby completing the proof of the upper bound in Proposition 4.3 .

Turning next to the lower bound on $f_{n, \ell, z}^{(+)}(y)$, we first truncate to $y \in$ $\left[\sqrt{\ell} /\left(2 r_{\ell}\right), \sqrt{\ell} 2 r_{\ell}\right]$ and restrict to $z^{\prime} \in\left[\frac{z}{2}, \frac{3 z}{2}\right]$ via the event $\mathcal{B}$. Then, taking $h_{\ell} \geq 2(T+1)^{\delta}$ for $T=T_{\epsilon}$ of Lemma 6.3, guarantees that

$$
3 h_{\ell} \geq \sup _{t \in[1, T+1] \cap\left[n^{\prime}-T, n^{\prime}\right]}\left\{2 \psi_{\ell}(t)\right\},
$$

for $\psi_{\ell}(\cdot) \geq h_{\ell}$ of (4.10). This in turn implies that

$$
D_{2 \psi, T} \subset D_{2 \psi} \bigcup\left(D_{3 h_{\ell}, 0, T+1}^{c} \cap D_{2 h_{\ell}, T}\right) \bigcup\left(D_{2 h_{\ell}, T} \cap D_{3 h_{\ell}, \widetilde{n}-T, n^{\prime}}^{c}\right),
$$

where $D_{3 h_{\ell}, T, T^{\prime}}^{c}$ denotes the event of the Brownian motion crossing below the linear barrier in the definition of $D_{3 h_{\ell}, T, T^{\prime}}$, see below (6.17). See figure 4 for an illustration of these events. From (6.40) and the LHS of (6.33) we deduce 


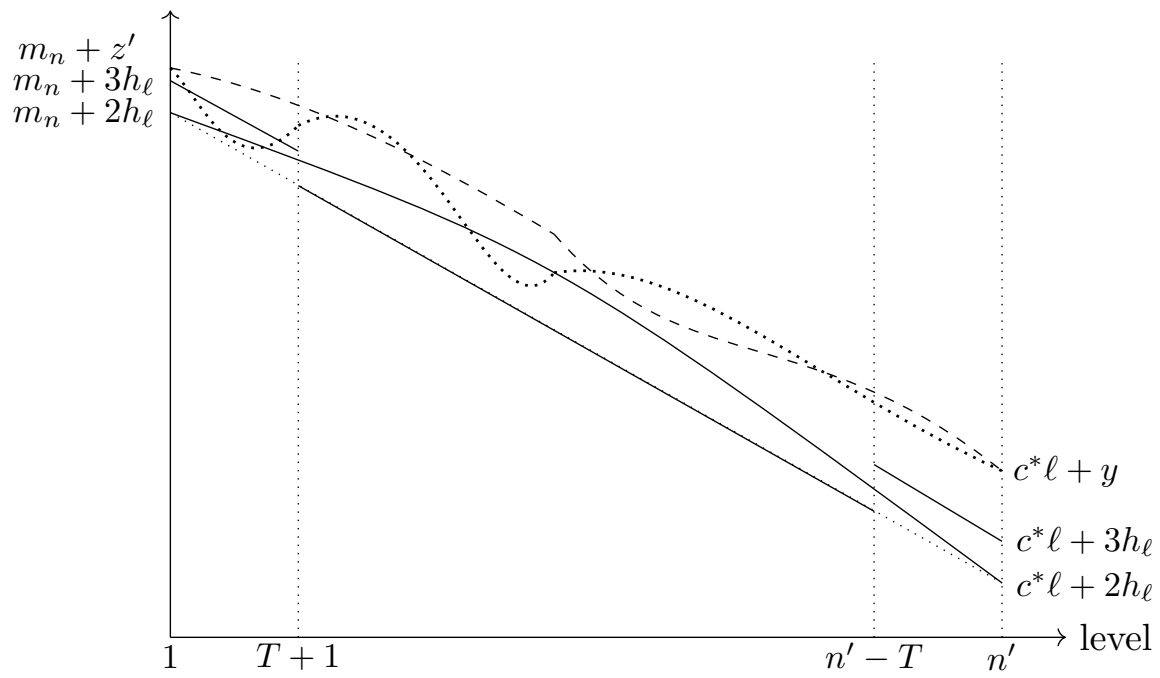

Figure 4. Depiction of the events $D_{2 \psi}$ (dashed curve) and $D_{3 h_{\ell}, 0, T+1}^{c} \cap D_{2 h_{\ell}, T}$ (dotted line).

by the union bound, that at $x=m_{n}+z^{\prime}, w=c_{*} \ell+y$, for all $\tilde{n}$ large enough

$$
\mathbf{q}_{\widetilde{n}}^{(+)}(x, w) \geq(1-\epsilon) \widetilde{\mathbf{q}}_{\tilde{n}, T}^{(+)}(x, w)-\widetilde{\mathbf{q}}_{\tilde{n}, T}^{\left(\downarrow 3 h_{\ell},+\right)}(x, w)-\widetilde{\mathbf{q}}_{\tilde{n}, T}^{\left(+, \downarrow 3 h_{\ell}\right)}(x, w),
$$

where $\widetilde{\mathbf{q}}_{\tilde{n}, T}^{\left(\downarrow 3 h_{\ell},+\right)}(x, w)$ and $\widetilde{\mathbf{q}}_{\tilde{n}, T}^{\left(+, \downarrow 3 h_{\ell}\right)}(x, w)$ are the probabilities of the events $D_{3 h_{\ell}, 0, T+1}^{c} \cap D_{2 h_{\ell}, T}$ and $D_{2 h_{\ell}, T} \cap D_{3 h_{\ell}, \widetilde{n}-T, n^{\prime}}^{c}$ under $\mathbb{P}_{x}^{W}\left(\cdot \mid W_{n^{\prime}}=w\right)$.

Proceeding to evaluating the latter terms, note that conditional on $(\bar{Z}, \bar{Y})$ and the given values of $W_{1}=x, W_{n^{\prime}}=w$, the events $D_{3 h_{\ell}, 0, T+1}, D_{2 h_{\ell}, T}$ and $D_{3 h_{\ell}, \widetilde{n}-T, n^{\prime}}$ are mutually independent. Thus, setting $y^{\prime}=w-\rho_{n} \ell \in[y, y+1]$ and assuming WLOG that $\left(\sqrt{\ell} / r_{\ell}\right) \wedge z \geq 8 h_{\ell}$, we have from (6.29), that

$$
\begin{aligned}
\mathbb{P}\left(D_{3 h_{\ell}, 0, T+1}^{c} \mid \bar{Z}, \bar{Y}\right) & =1-\mathbb{P}_{x \rightarrow W_{T+1}}^{[1, T+1]}\left(A_{\bar{\varphi}_{n}(t)+3 h_{\ell}}\right)=e^{-2\left(z^{\prime}+\rho_{n}-3 h_{\ell}\right)\left(\bar{Z}-3 h_{\ell}\right)_{+} / T}, \\
\mathbb{P}\left(D_{3 h_{\ell}, \widetilde{n}-T, n^{\prime}}^{c} \mid \bar{Z}, \bar{Y}\right) & =1-\mathbb{P}_{\left.W_{n^{\prime}-T} \rightarrow n^{\prime}-T, n^{\prime}\right]}\left(A_{\bar{\varphi}_{n}(t)+3 h_{\ell}}\right)=e^{-2\left(\bar{Y}-3 h_{\ell}\right)_{+}\left(y^{\prime}-3 h_{\ell}\right) / T} .
\end{aligned}
$$

Combining these identities with (6.32) and the inequality (6.41), we arrive at

$$
\begin{array}{r}
\mathbf{q}_{\tilde{n}}^{(+)}(x, w) \geq \mathbb{E}\left[\left(1-\epsilon-e^{-2\left(z^{\prime}+\rho_{n}-3 h_{\ell}\right)\left(\bar{Z}-3 h_{\ell}\right)_{+} / T}-e^{-2\left(y^{\prime}-3 h_{\ell}\right)\left(\bar{Y}-3 h_{\ell}\right)_{+} / T}\right)\right. \\
\left.\mathbb{P}_{\bar{Z}+\bar{\varphi}_{n}(T+1) \rightarrow \bar{Y}+\bar{\varphi}_{n}\left(n^{\prime}-T\right)}^{\left[T+1, n^{\prime}-T\right]}\left(A_{\bar{\varphi}_{n}(t)+2 h_{\ell}}\right)\right] .
\end{array}
$$

The first factor on the RHS is at least -2 and for all $\ell$ larger than some universal $\ell_{0}(\epsilon)$ it exceeds $(1-\epsilon)^{2}$ on the event $A:=\left\{\bar{Z} \wedge \bar{Y} \geq 4 h_{\ell}\right\}$. Setting $V:=\left(\bar{Z}-2 h_{\ell}\right)_{+}\left(\bar{Y}-2 h_{\ell}\right)_{+}$, we combine for the second term on the RHS the analog of identity (6.30) with the bound $1-e^{-a} \in\left[a-a^{2} / 2, a\right]$ on $\mathbb{R}_{+}$to arrive at

$$
(\widetilde{n}-2 T) \mathbf{q}_{\widetilde{n}}^{(+)}(x, w) \geq 2 \mathbb{E}\left[\left\{(1-\epsilon)^{2}-2 \mathbf{1}_{A^{c}}-\frac{2 V}{\widetilde{n}-2 T}\right\} V\right] .
$$


Utilizing (6.27), the uniform tail bounds one has on $\left(\bar{Z}-z^{\prime}, \bar{Y}-y\right)$ when $\widetilde{n} \rightarrow \infty$, for our truncated range of $z^{\prime}$ and $y$, followed by (6.37), we conclude that

$$
\begin{aligned}
& \varliminf_{z \rightarrow \infty} \varliminf_{n \rightarrow \infty}\left\{f_{n, \ell, z}^{(-)}(y)\right\} \geq \varliminf_{z \rightarrow \infty} \varliminf_{n \rightarrow \infty} \mathbb{E}\left[\mathbf{1}_{\mathcal{B}} \frac{e^{-c_{*} U_{s}}}{\sqrt{2} z} \frac{\widetilde{n}}{2} \mathbf{q}_{\widetilde{n}}^{(+)}\left(m_{n}+z^{\prime}, c_{*} \ell+y\right)\right] \\
& \geq(1-\epsilon)^{2}\left(y-3 h_{\ell}\right) \lim _{z, s \rightarrow \infty} \mathbb{E}\left[\mathbf{1}_{\mathcal{B}} \frac{\left(z^{\prime}-3 h_{\ell}\right)_{+}}{\sqrt{2} z} e^{-c_{*} U_{s}}\right] \geq(1-\epsilon)^{2}\left(y-3 h_{\ell}\right) .
\end{aligned}
$$

Plugging this into (6.26) and noting that for $\ell \rightarrow \infty$

$$
\frac{1}{\sqrt{\pi \ell}} \int_{\sqrt{\ell} /\left(2 r_{\ell}\right)}^{2 r_{\ell} \sqrt{\ell}} e^{c_{*} y} \widetilde{\gamma}_{\ell}(y) \frac{y-3 h_{\ell}}{\sqrt{1+y /\left(c_{*} \ell\right)}} d y \geq \alpha_{\ell}(1-\epsilon)
$$

we arrive at the RHS of (6.25), thereby completing the proof of Proposition 4.3 .

\section{REFERENCES}

[1] Y. Abe. Extremes of local times for simple random walks on symmetric trees arXiv:1603.09047, 2016.

[2] Y. Abe and M. Biskup. Exceptional points of two-dimensional random walks at multiples of the cover time. arXiv:1903.04045.

[3] E. Aïdékon. Convergence in law of the minimum of a branching random walk. Ann. Probab., 41:1362-1426, 2013.

[4] D. J. Aldous. Threshold limits for cover times. J. Th. Probab. 4:197-211, 1991.

[5] D. J. Aldous. Random walk covering of some special trees. J. Math. Anal. Appl., 157:271-283, 1991.

[6] L. P. Arguin. Extrema of log-correlated random variables: Principles and Examples. arXiv:1601.00582,

[7] D. Belius and N. Kistler. The subleading order of two dimensional cover times. Probab. Th. Rel. Fields, 167(1):461-552, 2017.

[8] D. Belius, J. Rosen and O. Zeitouni. Barrier estimates for a critical Galton-Watson process and the cover time of the binary tree. Ann. Inst. H. Poincare, Prob. Stat., $55: 127-154,2019$.

[9] D. Belius, J. Rosen and O. Zeitouni. Tightness for the cover time of $S^{2}$. arXiv:1711.02845, 2017.

[10] M. Biskup. Extrema of the two-dimensional discrete Gaussian free field. arXiv:1712.09972, 2017.

[11] A. Bovier. Gaussian processes on trees. From spin glasses to branching Brownian motion. Cambridge Studies in Advanced Mathematics, 163. Cambridge University Press, Cambridge, 2017.

[12] M. Bramson. Convergence of solutions of the Kolmogorov equation to traveling waves. Memoirs of Am. Math. Soc., 44(285):1-190, 1983.

[13] M. Bramson, J. Ding and O. Zeitouni. Convergence in law of the maximum of nonlattice branching random walk. Ann. Inst. H. Poincare, Prob. Stat. 52:1897-1924, 2016.

[14] M. Bramson and O. Zeitouni. Tightness for a family of recursion equations. Ann. Probab. 37:615-653, 2009.

[15] A. Cortines, O. Louidor and S. Saglietti A scaling limit for the cover time of the binary tree. arXiv:1812.10101, 2018.

[16] A. Dembo, Y. Peres, J. Rosen and O. Zeitouni. Cover times for Brownian motion and random walks in two dimensions. Ann. Math. 160:433-464, 2004.

[17] A. Dembo, Y. Peres, J. Rosen and O. Zeitouni. Late points for random walks in two dimensions. Ann. Probab. 34:219-263, 2006. 
[18] J. Ding. Asymptotics of cover times via Gaussian free fields: Bounded-degree graphs and general trees. Ann. Probab. 42:464496, 2014.

[19] J. Ding, J. Lee, and Y. Peres. Cover times, blanket times, and majorizing measures. Ann. Math. 175:14091471, 2012.

[20] J. Ding and O. Zeitouni. A sharp estimate for cover times on binary trees. Stoch. Proc. Appl. 122:2117-2133, 2012.

[21] N. Eisenbaum, H. Kaspi, M. B. Marcus, J. Rosen, and Z. Shi. A Ray-Knight theorem for symmetric Markov processes. Ann. Probab. 28:1781-1796, 2000.

[22] Y. Hu and Z. Shi. Minimal position and critical martingale convergence in branching random walks, and directed polymers on disordered trees. Ann. Probab. 37:742-789, 2009.

[23] N. Kistler. Derrida's random energy models. From spin glasses to the extremes of correlated random fields. Correlated random systems: five different methods, 71-120, Lecture Notes in Math., 2143, CIRM Jean-Morlet Ser., Springer, Cham, 2015.

[24] S. P. Lalley and T. Selke. A conditional limit theorem for the frontier of a branching Brownian motion. Ann. Probab. 15:10521061, 1987.

[25] J. Lehec. Cover times and generic chaining. J. Appl. Probab. 51: 247-261, 2014.

[26] P. C. Matthews Covering problems for Markov chains. Ann. Probab. 16:1215-1228, 1988.

[27] M. R. Schmidt. A simple proof of the DPRZ-theorem for 2D cover times. arXiv:1805.09744, 2018. To appear, Ann. Probab.

[28] A. S. Sznitman. Topics in occupation times and Gaussian free fields. Zurich Lectures in Adv. Math. European Mathematical Society (EMS), Zrich, 2012.

[29] O. Zeitouni. Branching random walks and Gaussian fields. Probability and statistical physics in St. Petersburg, 437-471, Proc. Sympos. Pure Math., 91, Amer. Math. Soc., Providence, RI, 2016.

[30] A. Zhai. Exponential concentration of cover times. Elec. J. Probab. 23, paper 32, 2018.

Amir Dembo

Department of Mathematics \& Department of Statistics

Stanford University, Stanford, CA 94305

adembo@stanford.edu

Jay Rosen

Department of Mathematics

College of Staten Island, CUNY

Staten Island, NY 10314

jrosen30@optimum.net

Ofer Zeitouni

Faculty of Mathematics, Weitzmann Institute and

Courant Institute, NYU

Rehovot 76100, Israel and NYC, NY 10012

ofer.zeitouni@weizmann.ac.il 